\title{
Effect of carbon on the microstructure evolution and mechanical properties of low Si-containing centrifugal casting $20 \mathrm{Cr} 32 \mathrm{Ni} 1 \mathrm{Nb}$ alloy
}

Jingbo Yan ${ }^{1,2}$, Yuefeng $\mathrm{Gu}^{2}$, Yingying Dang ${ }^{1}$, Xinbao Zhao ${ }^{1}$, Jintao $\mathrm{Lu}^{1}$, Yong Yuan ${ }^{1}$, Zhen Yang ${ }^{1}$, Hongfei Yin ${ }^{1}$

1. Xi'an Thermal Power Research Institute, Xingqing Road 136, Xi'an, Shaanxi 710032, China

2. High Temperature M aterials Unit, National Institute for Materials Science (NIM S), 12-1 Sengen, Tsukuba, Ibaraki 305-0047, Japan

Correspondence address:

Dr. Jingbo Yan

Address:

Xi'an Thermal Power Research Institute

Xingqing Road 136

Xi'an, Shaanxi 710032

China

E-mail: yf625oscar@163.com

Tel: +81-080-7961-7170

Fax: +81-029-859-2501 
Abstract: The dendrite structure, which is formed during centrifugal casting process, plays an important role in the fracture behavior of 20Cr32Ni1Nb alloy. The large primary carbide facilitates the crack nucleation and propagation along the dendrite interface during high temperature deformation. The secondary NbC precipitates near the dendrite interface during aging at $1120^{\circ} \mathrm{C}$, while the major precipitates change to secondary $\mathrm{Cr}_{23} \mathrm{C}_{6}$ when aging at lower temperature. These secondary carbides effectively strengthen the dendrite interface, which is probably due to inhibiting the crack propagation and decreasing the stress concentration. However, the benefit of the secondary carbide reduce when the precipitation free zone form aging at high temperature. Increasing $\mathrm{C}$ content enhances the precipitation tendency of $\mathrm{Cr}_{23} \mathrm{C}_{6}$, and facilitates the coalescing of the carbide at elevated temperature. As a result, the cracks can easily nucleate at the interface between $\mathrm{NbC}$ and $\mathrm{Cr}_{23} \mathrm{C}_{6}$, and then propagate along the large chromium carbide during deformation. However, the small chromium carbide precipitates form at lower aging temperature, and thus strengthens the alloy effectively.

Keywords: Fracture; Precipitation; Carbide; Crack; Mechanical properties 


\section{Introduction}

For recent years, $20 \mathrm{Cr} 32 \mathrm{Ni1} \mathrm{Nb}$ alloy has been considered as the most potential candidate material to be used as the reform tube in the petrochemical industry ${ }^{[1]}$. It is usually processed by centrifugal casting method, possessing columnar austenitic grain with $\mathrm{MX}$ carbide $(\mathrm{NbC})$ distributing at grain boundary and dendrite interface ${ }^{[2]}$. Generally, the $\mathrm{NbC}$ has been considered as the major strengthening phase in the alloy, and its strengthening effect achieves maximum when the $\mathrm{Nb} / \mathrm{C}$ is in stoichiometry ratio ${ }^{[3]}$. However, the $\mathrm{NbC}$ was reported to convert into $\mathrm{G}$ phase ${ }^{[4]}\left(\mathrm{Ni}_{16} \mathrm{Nb}_{6} \mathrm{Si}\right.$ ) during short aging at $700-850{ }^{\circ} \mathrm{C}^{[5-9]}$, leading to the fracture of $20 \mathrm{Cr} 32 \mathrm{Ni} 1 \mathrm{Nb}$ alloy at the early stage during service ${ }^{[10-14]}$. In recent years, some works proved that the $\mathrm{G}$ phase formation can be effectively inhibited when Si content has been restricted within low level ${ }^{[15,16]}$. However, the service performance of low Si-containing $20 \mathrm{Cr} 32 \mathrm{Ni} 1 \mathrm{Nb}$ alloy is still unclear.

On the other hand, there is a large difference on the microstructure characterization between the 20Cr32Ni1Nb alloy and the traditional reform tube materials (HP40 and HK40 alloy). The major difference is the low carbon content in $20 \mathrm{Cr} 32 \mathrm{Ni} 1 \mathrm{Nb}$ alloy, which leads to the much lower volume fraction of the carbide ${ }^{[17]}$. It has been extensively reported that the network chromium carbide develops at the grain boundary of HP40 and HK40 alloy, strengthening the alloy by inhibiting the grain boundary sliding during alloy deforming at elevated temperature ${ }^{[18,19]}$. By comparing, the $\mathrm{Cr}_{23} \mathrm{C}_{6}$ carbide in the 20Cr32Ni1Nb alloy usually form during service, precipitating at both the grain interior and boundary in fewer amount ${ }^{[20]}$. The absence of chromium carbide in the cast $20 \mathrm{Cr} 32 \mathrm{Ni} 1 \mathrm{Nb}$ alloy is mainly because of the $\mathrm{Nb} / \mathrm{C}$ ratio satisfies as below ${ }^{[21]}$ :

$[\mathrm{Nb}] /([\mathrm{C}]+[\mathrm{N}]) \cong 7.7$

Here, $[X]$ is the weight percentage of the $X$ element in the alloy. Nevertheless, this material possesses competitive strength at elevated temperature, and its superior ductility ensures the higher fatigue fracture resistance $^{[22]}$. This indicates that the carbide in the 20Cr32Ni1Nb alloy strengthening in a more effective way, probably because carbide distribution or morphology plays a more important role in their strengthening effect. However, the literature referring to the strengthening mechanism of this material is yet unavailable, especially associating with the centrifugal structure.

In the present work, we investigated microstructure evolution of low Si-containing 20Cr32Ni1Nb alloy after aging at various temperatures, and discussed the effect of aging temperature on the mechanical properties of the materials. Some of the solution strengthening elements, such as tungsten and molybdenum, were induced for further improving the microstructure stability and alloy properties ${ }^{[23-25]}$. In addition, a higher carbon modified alloy, which has the lower $\mathrm{Nb} / \mathrm{C}$ ratio, was also involved in this research. By comparing the precipitation behavior of carbide during solidification and aging duration, the strengthening mechanism of carbide in the $20 \mathrm{Cr} 32 \mathrm{Ni1Nb}$ alloy was discussed in this work. 


\section{Experimental Procedure}

Two grades of 20Cr32Ni1Nb alloys were involved in this investigation, and both of them were prepared by centrifugal casting method. The raw materials were melted in a 10-kg capacity medium frequency induction furnace, and poured into a horizontal centrifugal casting furnace when the melted alloy was super-heated to $1650{ }^{\circ} \mathrm{C}$. The ICP-OES was used for the chemical composition analyzing, and the results are shown in Table. 1 . Sample 1 is the low Si-containing $20 \mathrm{Cr} 32 \mathrm{Ni1Nb}$ alloy, with the $\mathrm{Nb} / \mathrm{C}$ ratio (in weight percentage) close to 7.7. Sample 2 possesses higher carbon content, which was designed to facilitate the M23C6 precipitation due to the low $\mathrm{Nb} / \mathrm{C}$ ratio.

The solution and aging treatment has been conducted for analyzing the microstructure evolution, and the detail parameters are shown in Table. 2. All the aged specimens were first homogenization at $1200{ }^{\circ} \mathrm{C}$ for 2 hours, and then aging at different temperature. The specimens for microstructure analyzing were taken from the same position of the casting ingot, selected at the position of $7 \mathrm{~mm}$ from the tube outer side, and sectioned into $12 \mathrm{~mm} \times 12 \mathrm{~mm} \times 3 \mathrm{~mm}$. After the aging procedure, the specimens were mounted in conductive resin and grounded on $\mathrm{SiC}$ sand paper up to 1000 \#, then polished by flannelette for less than $1 \mathrm{~min}$ and cleaned in acetone for $10 \mathrm{~min}$. XRD and EDX analyzing were conducted for investigating the phase constitution, and the back scattering electron microscopy (BSE) were used to investigate the microstructure evolution and element distribution. In addition, TEM specimen were prepared for analyzing the precipitates phase morphology and their orientation. They were mechanically thinned down to $50-100 \mu \mathrm{m}$ in thickness, followed by electro-polished in a solution of $45 \%$ acetic acid, $45 \% 2 \mathrm{n}$ butoxyethanol, and $10 \%$ perchloric acid at about $-10{ }^{\circ} \mathrm{C} / 30 \mathrm{~V}$.

The tensile test was conducted on both the cast and the heat treated materials, following the China national test standard GB/T 4338-2006. All the specimens were tested at the $750{ }^{\circ} \mathrm{C}$, and they were heated in the rate of $15{ }^{\circ} \mathrm{C} / \mathrm{min}$ from room temperature, held at the test temperature for $10 \mathrm{~min}$, and then started the test with the strain rate of $3 \times 10^{-4} \mathrm{~S}^{-1}$. SEM has been conducted for analyzing the detail of the fracture surface. The cross section of the fracture surface was prepared for analyzing the deformation mechanism of the alloy.

\section{Result and Discussion}

\subsection{Cr32Ni1Nb}

The microstructure of the centrifugal casting 20Cr32Ni1Nb alloy is shown in Fig. 1. The alloy is composed of austenitic matrix with so-called "Chinese Script" morphology carbide precipitates at dendrite interface and grain boundary (Fig. 1a). XRD analyzing confirms this carbide as NbC, as shown in Fig. 1b. It's usually been considered as the major strengthening phase in the 20Cr32Ni1Nb alloy, primarily because of their good stability during creep at elevated temperature.

The carbide precipitation behavior in various aging temperature is shown in Fig. 2. After aging at $1120{ }^{\circ} \mathrm{C}$ for 2 hours (HT1-1 alloy), some fine precipitates are observed close to the primary NbC, with the precipitation size rarely larger than $500 \mathrm{~nm}$ (Fig. 2a). According to the XRD analyzing result, these fine 
precipitates are deduced to be secondary NbC (Fig. 2d). This has been confirmed by and EDX elemental map analyzing, which show the small $\mathrm{Nb}$ rich particle precipitates within matrix (Fig. 3). The nucleation of $\mathrm{NbC}$ has frequently been observed in $20 \mathrm{Cr} 32 \mathrm{Ni} 1 \mathrm{Nb}$ alloy, and its precipitation orientation was reported as $[001]_{\mathrm{NbCl}} \|[001]_{\gamma}^{[22]}$. The alloy aging at $850{ }^{\circ} \mathrm{C}$ (HT2-1 alloy) exhibits some gray particles precipitate adjacent to the primary $\mathrm{NbC}$ (Fig. $2 \mathrm{~b}$ ). The precipitates show rectangle morphologies, and commonly possess the size within $100 \mathrm{~nm}$ in length. Because the particle is smaller than the EDX analyzing spot size, the results will be affected by the signal from the matrix. However, it can be deduced that the precipitate phase is $\mathrm{Cr}_{23} \mathrm{C}_{6}$ according to the XRD analyzing results. For further confirms the precipitate phase, TEM analyzing has been conducted in this work, as shown in Fig. 4a. Small precipitates carbide can be observed within the matrix, with the precipitation orientation of $[011]_{\mathrm{Cr} 23 \mathrm{C}} \|[011]_{\gamma}$. This is coincidence to others' work, which has reported the same orientation of the $\mathrm{Cr}_{23} \mathrm{C}_{6}$ during aging at $760^{\circ} \mathrm{C}^{[20]}$ and $900^{\circ} \mathrm{C}^{[5,16,24]}$, respectively. It can also be inferred that the precipitation orientation of carbide is not affected by the aging temperature. However, the precipitation of $\mathrm{Cr}_{23} \mathrm{C}_{6}$ shows fewer fractions when aging at $750{ }^{\circ} \mathrm{C}$ (HT3-1 alloy). On the other hand, quite fewer amount of secondary NbC can be observed in both HT2-1 and HT3-1 alloys, further confirming that secondary $\mathrm{NbC}$ primarily precipitates during aging at $1120^{\circ} \mathrm{C}$. The precipitation orientation of $\mathrm{NbC}$ has also been confirmed as $[001]_{\mathrm{NbC}} \|[001]_{\mathrm{r}}$, as shown in Fig. 4b. All of these results show strong dependence of the carbide precipitation behavior on the aging temperature of 20Cr32Ni1Nb alloy. Moreover, no G phase has been detected in the specimen aging at various temperatures, probably because of the low Si content in the alloy. This is quite different from other's work, which shows $G$ phase precipitates within several hours, especially in the temperature range between $700-850{ }^{\circ} \mathrm{C}^{[26]}$.

Tensile test at $750{ }^{\circ} \mathrm{C}$ has been conducted for investigating the mechanical properties of the materials. Compared with the commercial materials (CT15C), the higher strength and slightly lower ductility of 20Cr32Ni1Nb alloy probably associate with the addition of tungsten and molybdenum. On the other hand, the results show that the aging temperature has great effect on both the strength and the ductility (Fig. 5). Short aging at $1120^{\circ} \mathrm{C}$ doesn't seem to show obvious effect on the alloy strength, while the yield strength and area reduction increase significantly after aging at lower temperatures. The HT2-1 alloy (aged at 850 ${ }^{\circ} \mathrm{C}$ ) gains the highest mechanical properties at $750{ }^{\circ} \mathrm{C}$, including both the yield strength and the area reduction. In addition, the decrease of the ductility, usually induced by the $G$ phase formation during aging at $700-850{ }^{\circ} \mathrm{C}^{[27]}$, is not observed in this work.

The comparison on the rupture surface has been made among the samples, and the dendrite structure can be observed in all the test specimens (Fig. 6). This indicates that the sliding at the dendrite interface take place during deformation, and probably plays an important role in the rupture of the material. This is in good agreement with Luis's work, who observed that the dendrite interface is the main crack path in 20Cr32Ni1Nb alloy ${ }^{[28]}$. However, the specimens with various aging methods show some different characteristics at the fracture surface. For the HT2-1 and HT3-1 alloys, heterogeneous deformation occurs and the largest deformation direction is approximately $30-45^{\circ}$ to the dendrite growth direction. Moreover, the dimple can be easily identified by the higher magnification observation of the secondary crack, which 
indicates that a larger sliding at dendrite interface is allowed before the fracture of the HT2-1 and HT3-1 alloy. This is quite different from the cast and HT1-1 alloys, which did not experience large deformation before rupture took place.

These phenomena have been confirmed by the cross-section of the fracture specimen, as shown in Fig. 7. The void can be found at the interface between the matrix and $\mathrm{NbC}$, as well as some microcrack in the large NbC particle. This demonstrates that the interface between primary $\mathrm{NbC}$ and matrix is the weak point which acts as the cracks nucleation site, and thus facilitates the dendrite sliding during deformation. Similar phenomenon has been observed by Kargarnejad, who found that the Nb-rich particle in the matrix is the preferred sites to generate the voids ${ }^{[29]}$. However, by compare to the cast and HT1-1 alloy, the subsurface of the HT2-1 and HT3-1 alloy experiences the significant deformation before the cracks propagation. This confirms a larger sliding at dendrite interface took place before crack propagation. According to the classical fracture theory, the threshold of crack propagation can be described as below ${ }^{[30}$, 31]:

$\mathrm{G}_{1}>\mathrm{G}_{1 \mathrm{C}}$

Here, $\mathrm{G}_{1}$ is the driving force energy of the crack propagation, which depends on the applied stress condition; G1c is the critical energy required for the crack propagation, which is associated with the inherent properties of the material. G1 and the G1c can be rough estimated as below:

$\mathrm{G}_{1}=\sigma^{2} \pi \mathrm{c} / \mathrm{E}$

$\mathrm{G}_{1 \mathrm{C}}=2\left(\gamma_{\mathrm{s}}+\gamma_{\mathrm{\rho}}\right)$

Here, $\sigma$ could be considered as the stress condition nearby the crack, $\mathrm{E}$ is the modulus of elasticity, $\mathrm{c}$ is the size of the micro-void, $\gamma_{s}$ is the surface energy of the material, $\gamma_{\rho}$ is the energy required for the crack to penetrate the strain field at the crack tip.

Thus, it can be inferred that the crack propagation primarily depends on the stress level and the strain field condition at the dendrite interface. On the other hand, extensive rod like void has been observed adjacent to the primary NbC in the HT2-1 alloy, and all of them grow toward the deformed direction. This indicates that the secondary $\mathrm{Cr}_{23} \mathrm{C}_{6}$ adjacent to the primary $\mathrm{NbC}$ has pinning the matrix during alloy deformation, and thus relieves the stress concentration at the $\mathrm{NbC/matrix} \mathrm{interface.} \mathrm{Similar} \mathrm{phenomenon}$ has been observed in the HT3-1 alloy, but the amount of the rod like void is in the less extent. In addition, $\mathrm{Cr}_{23} \mathrm{C}_{6}$ precipitates adjacent to primary $\mathrm{NbC}$ may have an effect on the strain field generation at the dendrite interface, which further inhibits the crack propagation during deformation. Although the small precipitate has also been detected in HT1-1 alloy, its positive effect can be eliminated for the formation of the precipitation free zone (PFZ) between the primary and secondary NbC.

\subsection{Cr32Ni1Nb with high C content}

Fig. 8 shows the microstructure analyzing results on the 20Cr32Ni1Nb alloy with higher carbon content. Compared with the 20Cr32Ni1Nb alloy, the carbon content increase leads to the directly 
formation of the primary $\mathrm{Cr}_{23} \mathrm{C}_{6}$ during solidification. This is primarily induced by the low $\mathrm{Nb} /(\mathrm{C}+6 / 7 \mathrm{~N})$ ratio, which facilitates the chromium carbide formation when the value is below $7.7^{[32]}$.

Increasing $\mathrm{C}$ content in $20 \mathrm{Cr} 32 \mathrm{Ni1Nb}$ alloy will lead to the different precipitation behaviors, and it is closely related to the aging temperature (Fig. 9). The material aging at $1120{ }^{\circ} \mathrm{C}$ (HT1-2 alloy) shows the carbide precipitates at grain boundary and dendrite interface, whose size can sometimes reach up to 2 $\mu \mathrm{m}$ in equivalent diameter (Fig. 9a). This is because the excess carbon was ejected and segregated at dendrite interface during the solidification ${ }^{[19,33]}$, leading to the supersaturated solution of carbon. As a comparison, large amounts of secondary carbide can be observed in the materials aged at $850{ }^{\circ} \mathrm{C}$ (HT2-2 alloy) and $750{ }^{\circ} \mathrm{C}$ (HT3-2 alloy), mainly precipitates at dendrite interface with the relatively smaller size (Fig. 9b, c). This carbide has been confirmed as the $\mathrm{Cr}_{23} \mathrm{C}_{6}$ by EDX elemental analysis, as shown in Fig. 10. It is quite different from the precipitation behavior of $20 \mathrm{Cr} 32 \mathrm{Ni1} \mathrm{Nb}$ alloy, which exhibits the nose temperature of $\mathrm{Cr}_{23} \mathrm{C}_{6}$ precipitation at approximately $850{ }^{\circ} \mathrm{C}$. As the carbon content increases, larger amount of $\mathrm{Cr}_{23} \mathrm{C}_{6}$ can be observed after aging at $750{ }^{\circ} \mathrm{C}$, indicating the nose temperature decreased accompanied with the composition modification. This phenomenon can be explained from the aspect of thermodynamics, and the nucleation tendency of the carbide can be decided as following:

$\Delta \mathrm{G}=\pi \mathrm{r}^{3}(\Delta \mathrm{G} v+\Delta \mathrm{G} \varepsilon)+4 \pi \mathrm{r}^{2} \gamma_{\alpha \beta}<0$

Here, $\Delta \mathrm{G}$ is the Gibbs energy change for the carbide precipitation; $\Delta \mathrm{G} v$ and $\Delta \mathrm{G} \varepsilon$ are unit chemical energy and strain energy change during the precipitation, respectively; $r$ is the radius of the carbide particle; $\gamma_{\alpha \beta}$ is the interface energy between the carbide and matrix. Thus, the carbide will precipitate when the $(\Delta \mathrm{G} v+\Delta \mathrm{G} \varepsilon)<0$. In Eq. 5 , all the $\Delta \mathrm{G} v, \Delta \mathrm{G} \varepsilon$ and $\gamma_{\alpha \beta}$ are determined by the aging temperature. Thus, it could be inferred that, as for the $20 \mathrm{Cr} 32 \mathrm{Ni1Nb}$ alloy, the carbide precipitation reached the maximum at about $850^{\circ} \mathrm{C}$, as well as the $\left.\right|_{T \cong 850}=0$. However, the driving force of the precipitation, $\Delta \mathrm{G} v$, is strongly affected by the super cooling degree, which can be calculated as:

$\Delta \mathrm{G}_{v}=\left(\mathrm{T}_{\mathrm{c}}-\mathrm{T}\right) \Delta \mathrm{S}=\Delta \mathrm{T} \Delta \mathrm{S}$

Here, $T_{c}$ is the starting temperature of the precipitation; $T$ is the aging temperature; $\Delta S$ is the entropy change during precipitation. Thus, the $\Delta \mathrm{G}$ v greatly increased in the high C-adopted 20Cr32Ni1Nb alloy, mainly because the starting precipitation temperature $\left(T_{c}\right)$ significantly increased. Although the high carbon content may also increases the $\Delta \mathrm{G} \varepsilon$ and $\gamma \alpha \beta$, but it is relatively small compared with the increase of $\Delta \mathrm{G} v$ at the very beginning of the precipitation. Thus, the temperature should be lower for the higher $\Delta \mathrm{G} \varepsilon$ and $\gamma_{\alpha \beta}$ in the high C-adopted 20Cr32Ni1Nb system, and thus the nose temperature should decrease. As a comparison, when the alloy aging at higher temperature, the $\Delta \mathrm{G} v$ is too small that the chromium carbide cannot precipitate from the matrix directly. Thus, the carbide precipitates at the interface between $\mathrm{NbC}$ and matrix, which needs much lower $\Delta \mathrm{G} \varepsilon$ and $\gamma_{\alpha \beta}$ during the carbide nucleation. At the same time, the rapidly diffusion of the atom at elevated temperature permits the fast coalesce of the chromium carbide, and thus results in the formation of large $\mathrm{Cr}_{23} \mathrm{C}_{6}$ adjacent to the primary NbC in HT1-1 alloy. In addition, secondary $\mathrm{NbC}$ particles have been observed nearby the dendrite interface, and keep their size in various aging temperatures. 
The tensile test result is shown in Fig. 11. It has been confirmed again that the aging temperature has great effect on the yield strength and alloy ductility, and the maximum ductility was achieved after aging at $850{ }^{\circ} \mathrm{C}$. Compared with $20 \mathrm{Cr} 32 \mathrm{Ni} 1 \mathrm{Nb}$ alloy, the yield strength of high C-adopted alloy is relatively lower after aging at $850{ }^{\circ} \mathrm{C}$, probably because of its relatively lower tungsten content ${ }^{[34]}$. However, the strength of high C-adopted alloy shows kind of different tendency, and the highest value was obtained after $750{ }^{\circ} \mathrm{C}$ aging. This is probably because of the excess precipitation of carbide, which is accompanied with the decrease of alloy ductility.

The observation of the fracture surface confirmed the difference rupture mechanism of the materials (Fig. 12). All high C content 20Cr32Ni1Nb alloys show the dendrite structure characteristics on the fracture surface, indicating the weakness at the dendrite interface. However, the HT2-2 and HT3-2 alloys gain higher fraction of transgranular fracture, as well as the relatively larger deformation along the dendrite growth direction. Moreover, dimple can easily be found at the dendrite interface of the alloy aged at lower temperatures.

The cross-section of the fracture subsurface has been observed by BSE, and the results show great effect of the carbide size on the crack propagation behavior (Fig. 13). It can be seen that the void is prone to nucleate at the interface between large $\mathrm{Cr}_{23} \mathrm{C}_{6}$ particle and primary $\mathrm{NbC}$, and usually develops into relatively large size. The micro-cracks also have been observed within the large $\mathrm{Cr}_{23} \mathrm{C}_{6}$ particle in HT1-1 alloy, showing a convenient path for the cracks propagation. According to Eq. 4, the threshold of the crack prolongation depends on both the $\gamma_{s}$ and the $\gamma_{\rho}$. Due to the poor ductility of both $\mathrm{Cr}_{23} \mathrm{C}_{6}$ and $\mathrm{NbC}$, the size of the strain field $\left(\gamma_{\rho}\right)$ should be very small, which demonstrates that $\gamma_{\rho}$ could be neglected in this case, and thus the crack propagation resistance becomes smaller. This probably responds to the low properties of HT1-2 alloy at the test temperature. The void between two kinds of carbide was also found in HT2-2 and HT3-2 alloys, but such kind of void rarely forms beside small $\mathrm{Cr}_{23} \mathrm{C}_{6}$ particle near the primary NbC.

\section{Conclusion}

1. The aging temperature greatly affects the precipitation behavior of the secondary carbide. NbC will precipitate near the dendrite interface after the 2 hours of aging at $1120{ }^{\circ} \mathrm{C}$, as well as the PFZ developed between the primary and the secondary $\mathrm{NbC}$. On the other hand, small $\mathrm{Cr}_{23} \mathrm{C}_{6}$ achieved the greatest precipitation tendency at $850{ }^{\circ} \mathrm{C}$, adjacent to the primary $\mathrm{NbC}$ carbide.

2. The distribution of the secondary carbide affects the mechanical properties of 20Cr32Ni1Nb alloy at elevated temperatures. Small secondary carbide precipitates at dendrite interface significantly improve the yield strength and the ductility of the material, mainly because the crack propagation at the interface between primary $\mathrm{NbC}$ and matrix was effectively inhibited. However, this positive effect will be eliminated when the PFZ developed between the primary and the secondary carbide.

3. Increasing the carbon content in the 20Cr32Ni1Nb affects the microstructure and the mechanical properties significantly. The primary $\mathrm{Cr}_{23} \mathrm{C}_{6}$ forms at the dendrite interface of the cast high C- 
20Cr32Ni1Nb alloy. Aging at $1120{ }^{\circ} \mathrm{C}$ facilitates the precipitation of large $\mathrm{Cr}_{23} \mathrm{C}_{6}$ adjacent to the $\mathrm{NbC}$, which acts as the crack propagation path during alloy deformation. Aging at lower temperature facilitates the disperse distribution of small secondary $\mathrm{Cr}_{23} \mathrm{C}_{6}$ at dendrite interface. However, once the $\mathrm{Cr}_{23} \mathrm{C}_{6}$ growth into large size, it plays an important role in the crack nucleation during deformation, and thus brings negative effect on the ductility of the materials.

\section{Acknowledge}

We great appreciate the Natural Science Fund Committee, and this work was financially supported by National Science Foundation of China (Nos. 51301130 and 51301131).

\section{Reference}

[1] A. Energy, in, API, 2011, pp. 942-A.

[2] D.M. Knowles, C.W. Thomas, D.J. Keen, Q.Z. Chen, International Journal of Pressure Vessels and Piping, 81 (2004) 499-506.

[3] J. Wadsworth, J.H. Woodhead, S.R. Keown, M etal Science, 10 (1976) 342-348.

[4] H.J. B. Jun, F.L. Versnyder, Nature, 178 (1956) 208-209.

[5] G.D. Barbabela, L.H. de Almeida, T.L. da Silveira, I. Le M ay, M aterials Characterization, 26 (1991) 1-7.

[6] G.D. Barbabela, L.H. Dealmeida, T.L. Dasilveira, I. Lemay, Materials Characterization, 26 (1991) $193-$ 197.

[7] G.D.D. Soares, L.H. Dealmeida, T.L. Dasilveira, I. Lemay, Materials Characterization, 29 (1992) $387-$ 396.

[8] D.J. Powell, R. Pilkington, D.A. M iller, Acta M etallurgica, 36 (1988) 713-724.

[9] J. Yan, Y. Gu, J. Lu, Materials Science and Technology, (2014).

[10] J. Hoffman, in: NACE International, Corrosion 2000, 2000, pp. no. 512.

[11] R. Colwell, J. Hoffman, in: NACE International, 1998, pp. no. 423.

[12] L.S. M onobe, C.G. Schön, International Journal of Pressure Vessels and Piping, 86 (2009) 207-210.

[13] J.C. Guimarães Teixeira, G.V. Peixoto Donato, L.N. Júnior, C.B. Eckstein, in: World Petroleum Congress Proceedings, 2006.

[14] S. Shi, J.C. Lippold, J. Ramirez, Welding Journal (M iami, Fla), 89 (2010) 210S-217S.

[15] S. Zhao, X. Xie, G.D. Smith, S.J. Patel, Materials \& Design, 27 (2006) 1120-1127.

[16] R.A. Pedro Ibañez, G.D. de Almeida Soares, L.H. de Almeida, I. Le May, Materials Characterization, 30 (1993) 243-249.

[17] S. Shi, J.C. Lippold, M aterials Characterization, 59 (2008) 1029-1040.

[18] I.A. Sustaita-Torres, S. Haro-Rodríguez, M .P. Guerrero-M ata, M. de la Garza, E. Valdés, F. DeschauxBeaume, R. Colás, M aterials Chemistry and Physics, 133 (2012) 1018-1023. 
[19] X.Q. Wu, H.M. Jing, Y.G. Zheng, Z.M. Yao, W. Ke, Z.Q. Hu, M aterials Science and Engineering: A, 293 (2000) 252-260.

[20] Q.Z. Chen, C.W. Thomas, D.M . Knowles, M aterials Science and Engineering: A, 374 (2004) 398-408.

[21] M .P. Dewar, A.P. Gerlich, M etall. Mater. Trans. A-Phys. Metall. Mater. Sci., 44A (2013) 627-639.

[22] M. Dewar, in: Department of Chemical and Materials Engineering, University of Alberta, Edmonton, Alberta, 2012.

[23] B. Piekarski, M aterials Characterization, 47 (2001) 181-186.

[24] L.H. de Almeida, A.F. Ribeiro, I. Le M ay, Materials Characterization, 49 (2003) 219-229.

[25] J. Yan, Y. Gao, F. Yang, C. Yao, Z. Ye, D. Yi, S. Ma, Materials Science and Engineering: A, 529 (2011) 361-369.

[26] R.C. Ecob, R.C. Lobb, V.L. Kohler, J. M ater. Sci., 22 (1987) 2867-2880.

[27] J. Hoffman, J. Magnan, in: NACE International, Corrosion 2003, 2003, pp. no. 3469.

[28] L.S. M onobe, C.G. Schön, Journal of M aterials Research and Technology, 2 (2013) 195-201.

[29] S. Kargarnejad, V. Abbasi-Chianeha, Case Studies in Engineering Failure Analysis.

[30] G.R. Irwin, in: Fracturing of Metals, Proceedings of the ASM symposium on Fracturing of M etals, Cleveland, 1948, pp. 147-166.

[31] E. Orowan, in: Reports on Progress in Physics, 1949, pp. 185-232.

[32] S.R. Keown, F.B. Pickering, in: Creep Strength in Steel and High Temperautre Alloys, Metals Society Londres, London, 1974, pp. 134-143.

[33] F.C. Nunes, J. Dille, J.L. Delplancke, L.H. de Almeida, Scripta M aterialia, 54 (2006) 1553-1556.

[34] D.J. Crudden, A. M ottura, N. Warnken, B. Raeisinia, R.C. Reed, Acta M aterialia, 75 (2014) 356-370. 
Table. 1 Chemical composition of the materials

\begin{tabular}{|c|c|c|c|c|c|c|c|c|c|}
\hline \multirow{2}{*}{ Alloy } & \multicolumn{9}{|c|}{ Weight percentage of the element /wt.\% } \\
\hline & $\mathrm{Fe}$ & $\mathrm{Cr}$ & $\mathrm{Ni}$ & $\mathrm{C}$ & $\mathrm{W}$ & Mo & $\mathrm{Nb}$ & $\mathrm{Si}$ & $\mathrm{Mn}$ \\
\hline 1. $20 \mathrm{Cr} 32 \mathrm{Ni} 1 \mathrm{Nb}$ & Bal. & 22.51 & 33.28 & 0.10 & 2.86 & 0.95 & 0.77 & 0.14 & 0.99 \\
\hline 2. High C-20Cr32Ni1Nb & Bal. & 23.94 & 36.61 & 0.19 & 1.83 & 0.95 & 0.79 & 0.16 & 0.96 \\
\hline 20Cr32Ni1Nb Alloy* & Bal. & $19-22$ & $31-35$ & $0.07-0.16$ & & & $0.6-1.6$ & $1.2 \leq$ & $1.5 \leq$ \\
\hline
\end{tabular}

*: This composition refers to the ASTM: A 351 (Grade CT 15C) 
Table. 2 Aging parameters of alloy

\begin{tabular}{cc}
\hline & Aging parameters \\
\hline HT 1 & $1200{ }^{\circ} \mathrm{C} / 2.0 \mathrm{~h}(\mathrm{AC})+1120{ }^{\circ} \mathrm{C} / 2.0 \mathrm{~h}(\mathrm{AC})$ \\
HT 2 & $1200{ }^{\circ} \mathrm{C} / 2.0 \mathrm{~h}(\mathrm{AC})+850{ }^{\circ} \mathrm{C} / 18.0 \mathrm{~h}(\mathrm{AC})$ \\
HT 3 & $1200{ }^{\circ} \mathrm{C} / 2.0 \mathrm{~h}(\mathrm{AC})+750{ }^{\circ} \mathrm{C} / 30.0 \mathrm{~h}(\mathrm{AC})$ \\
\hline
\end{tabular}




\section{Table Captions}

Table. 1 Chemical composition of the materials

Table. 2 Aging parameters of alloy 


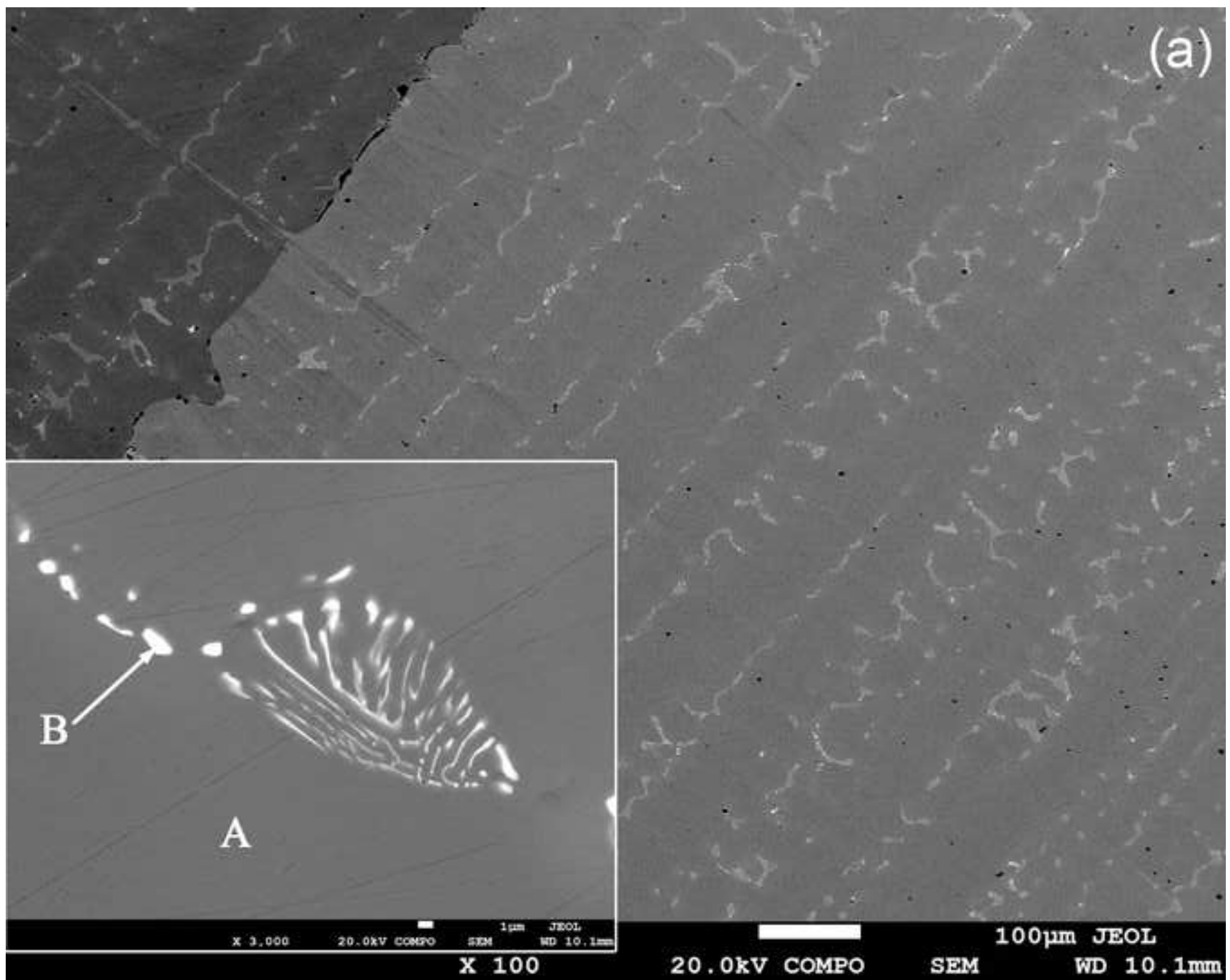




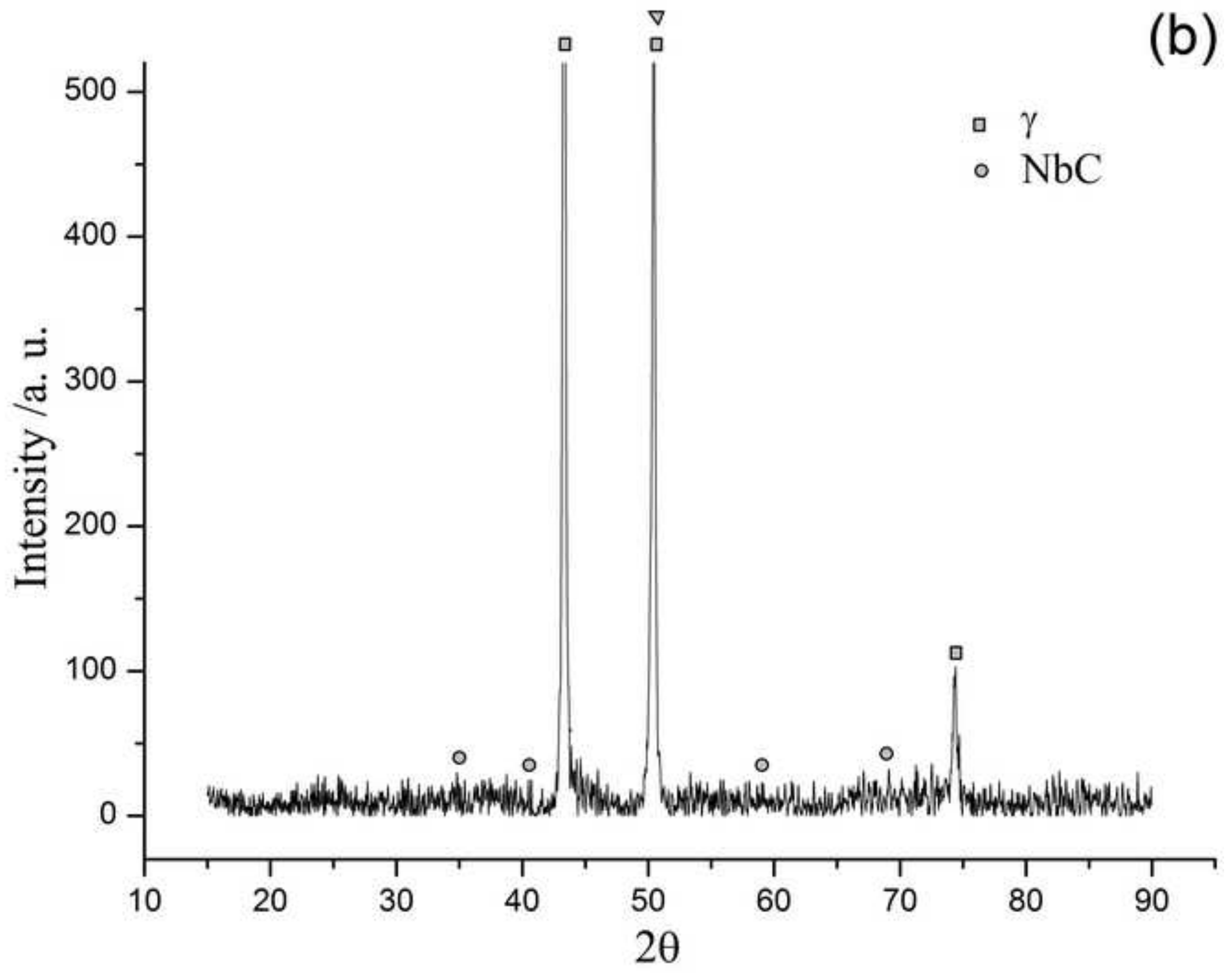




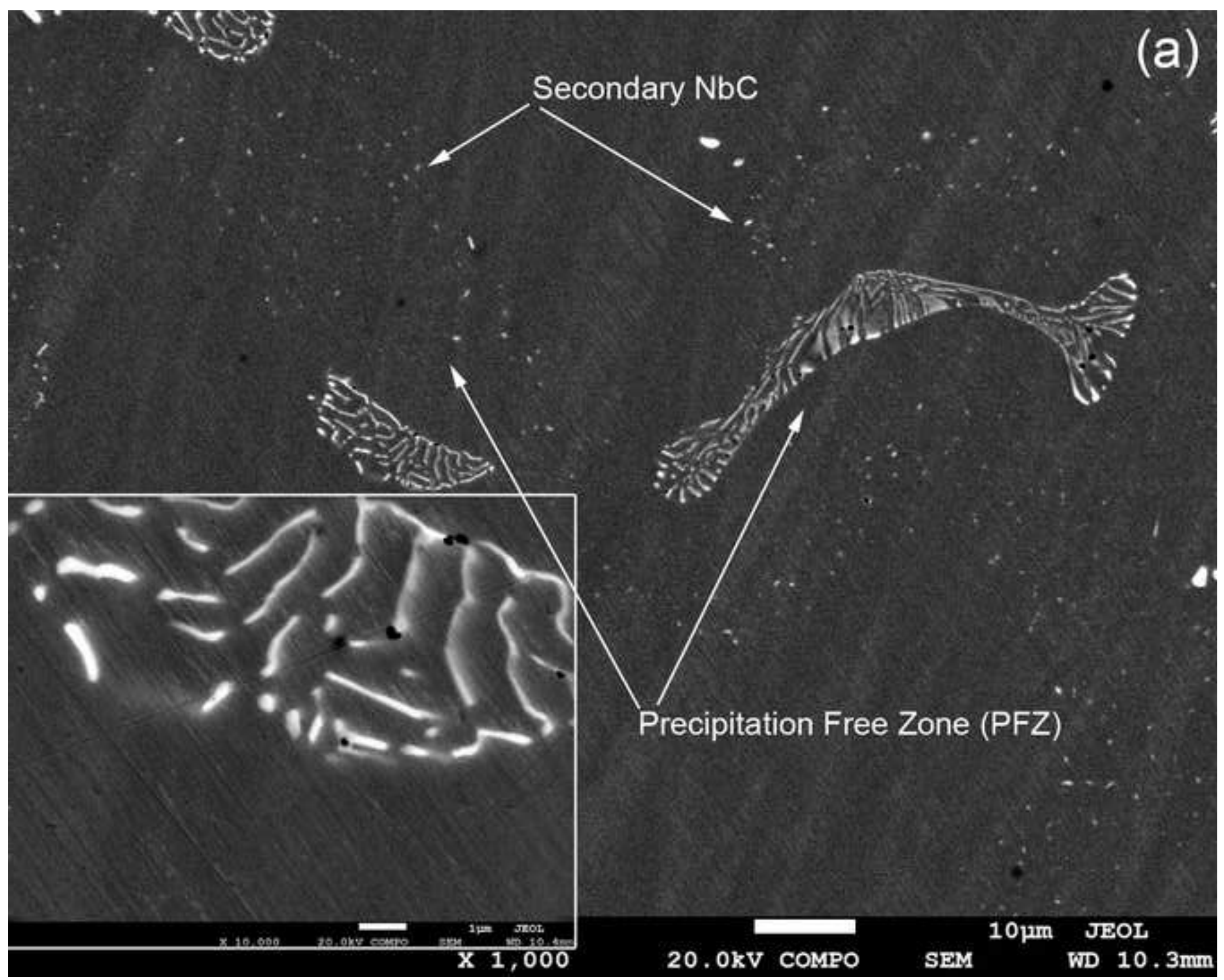




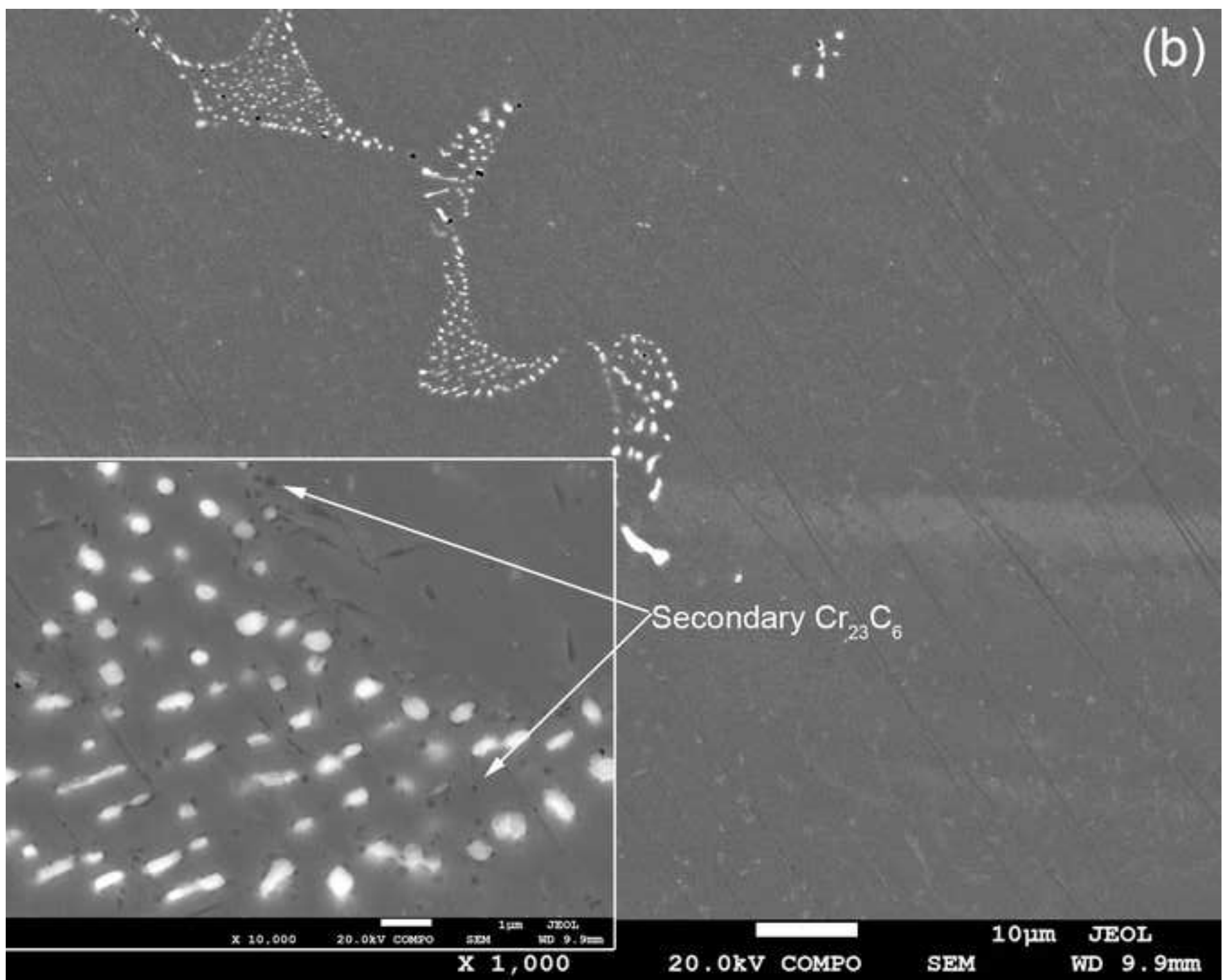

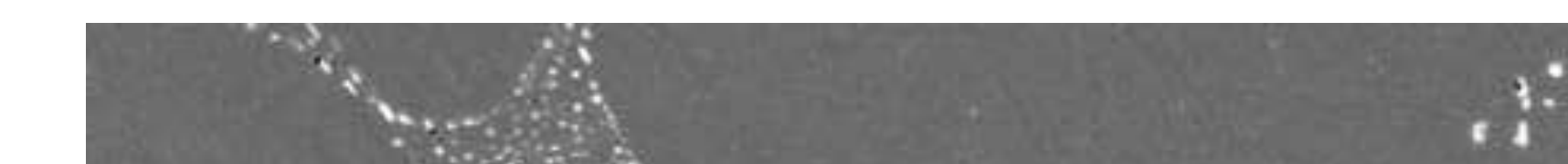

(b) 


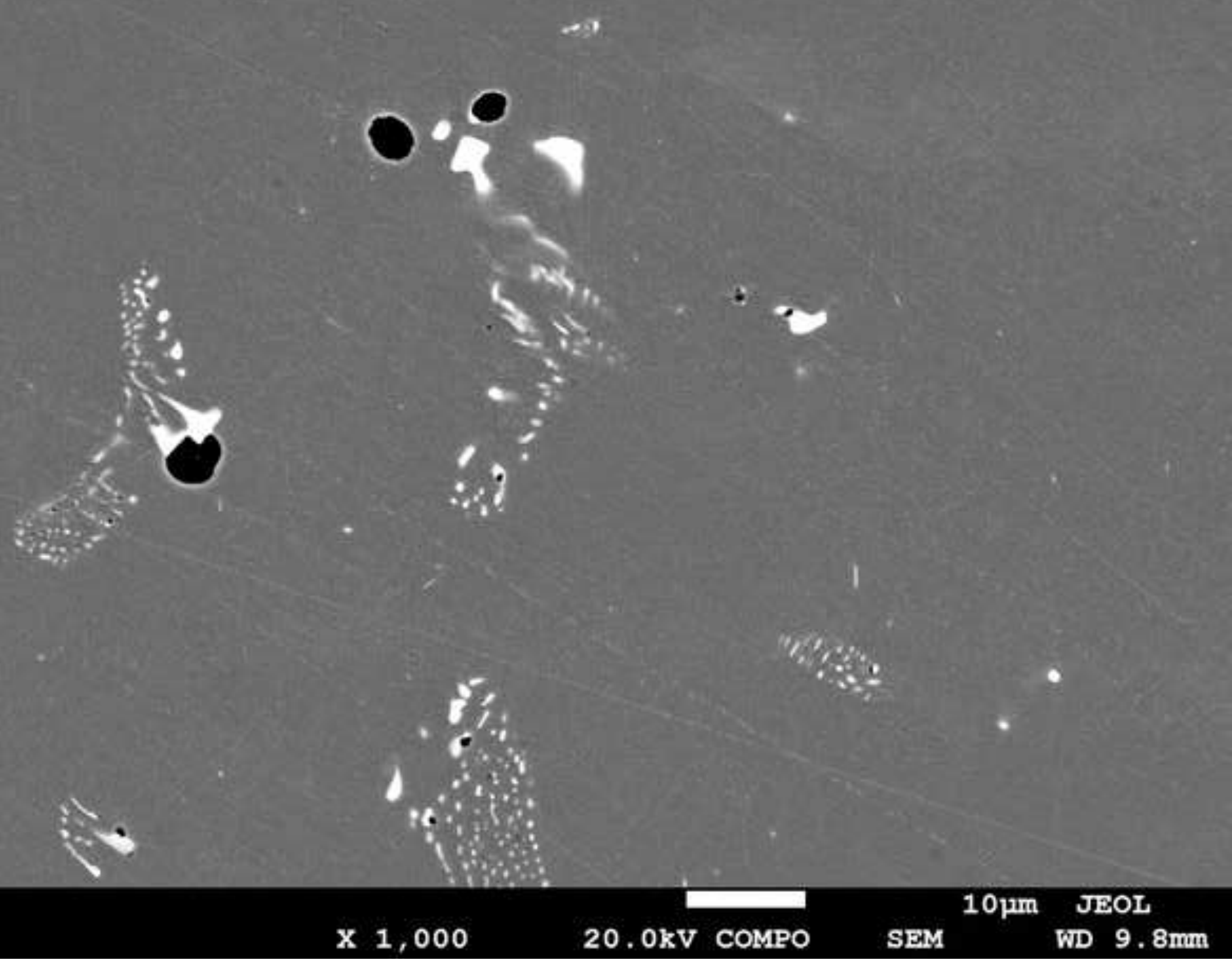




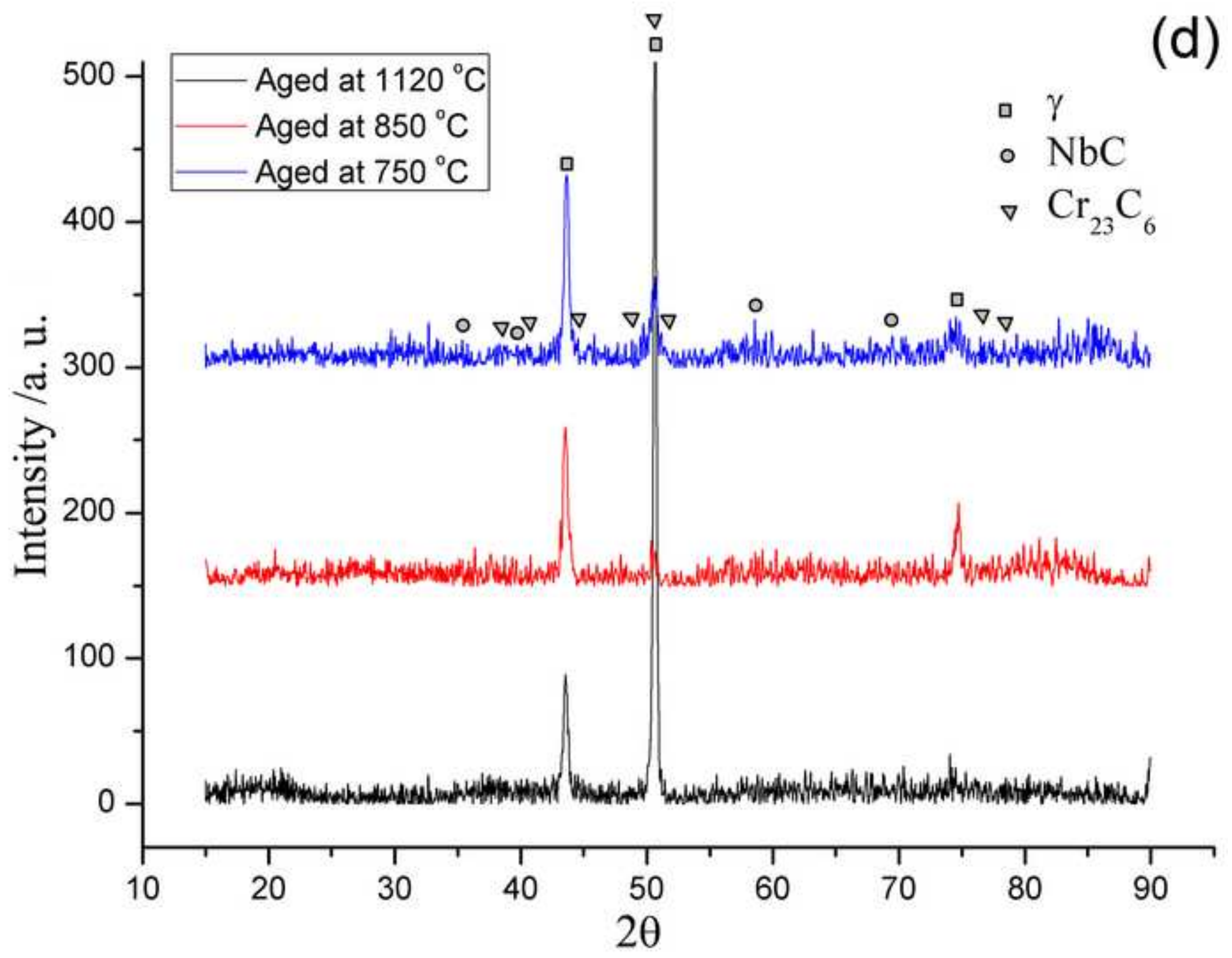



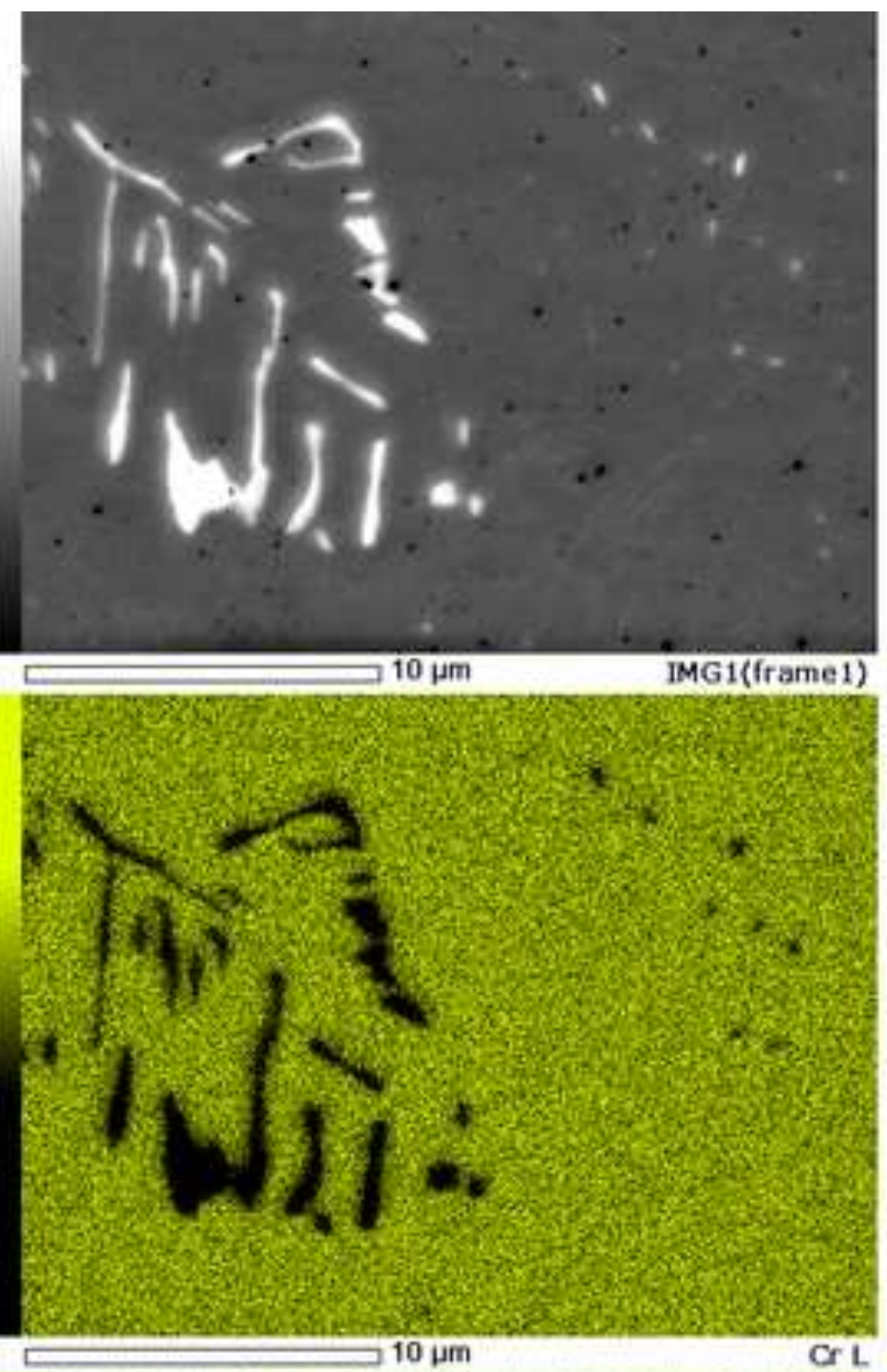

Cr L
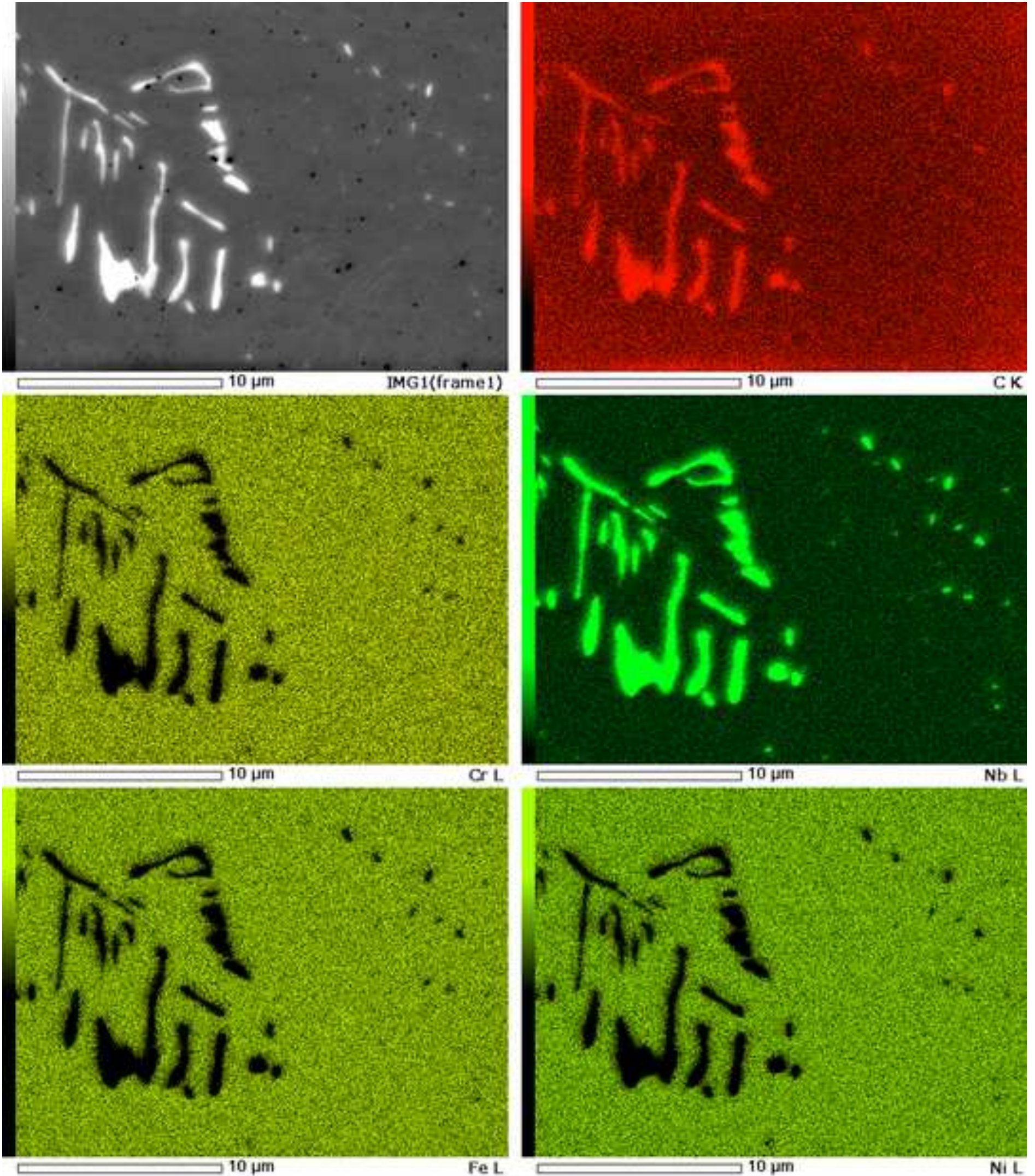


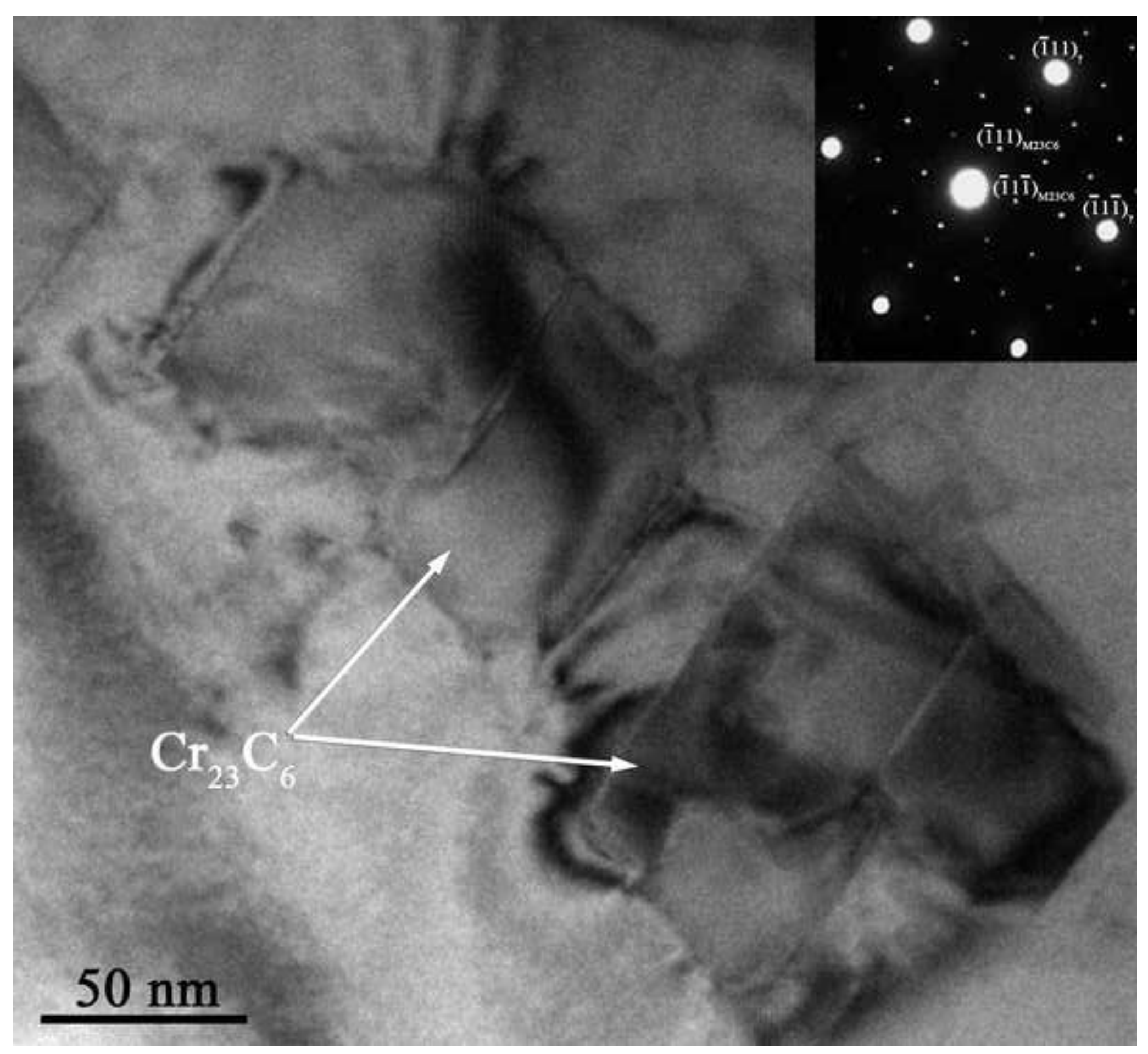




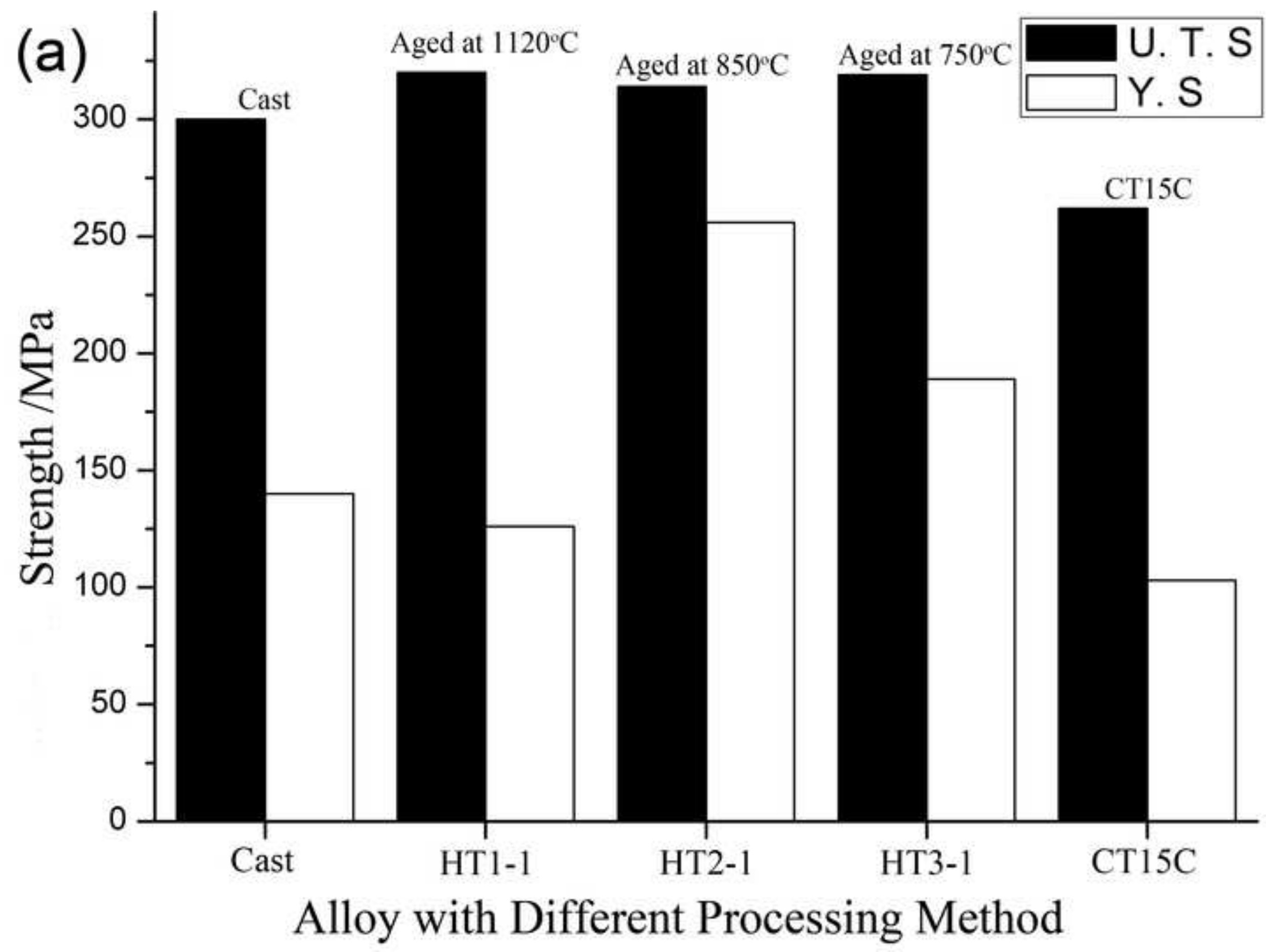




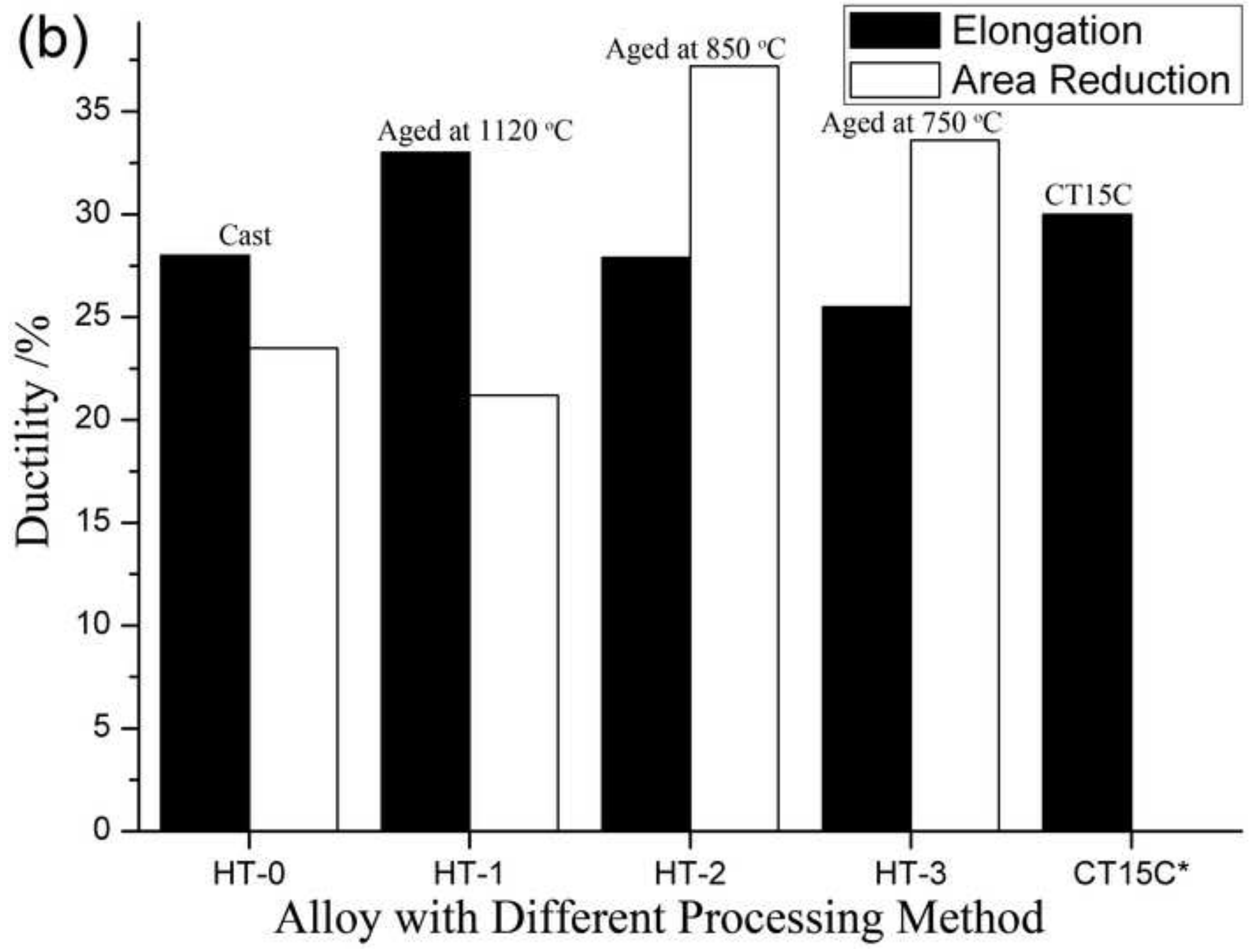




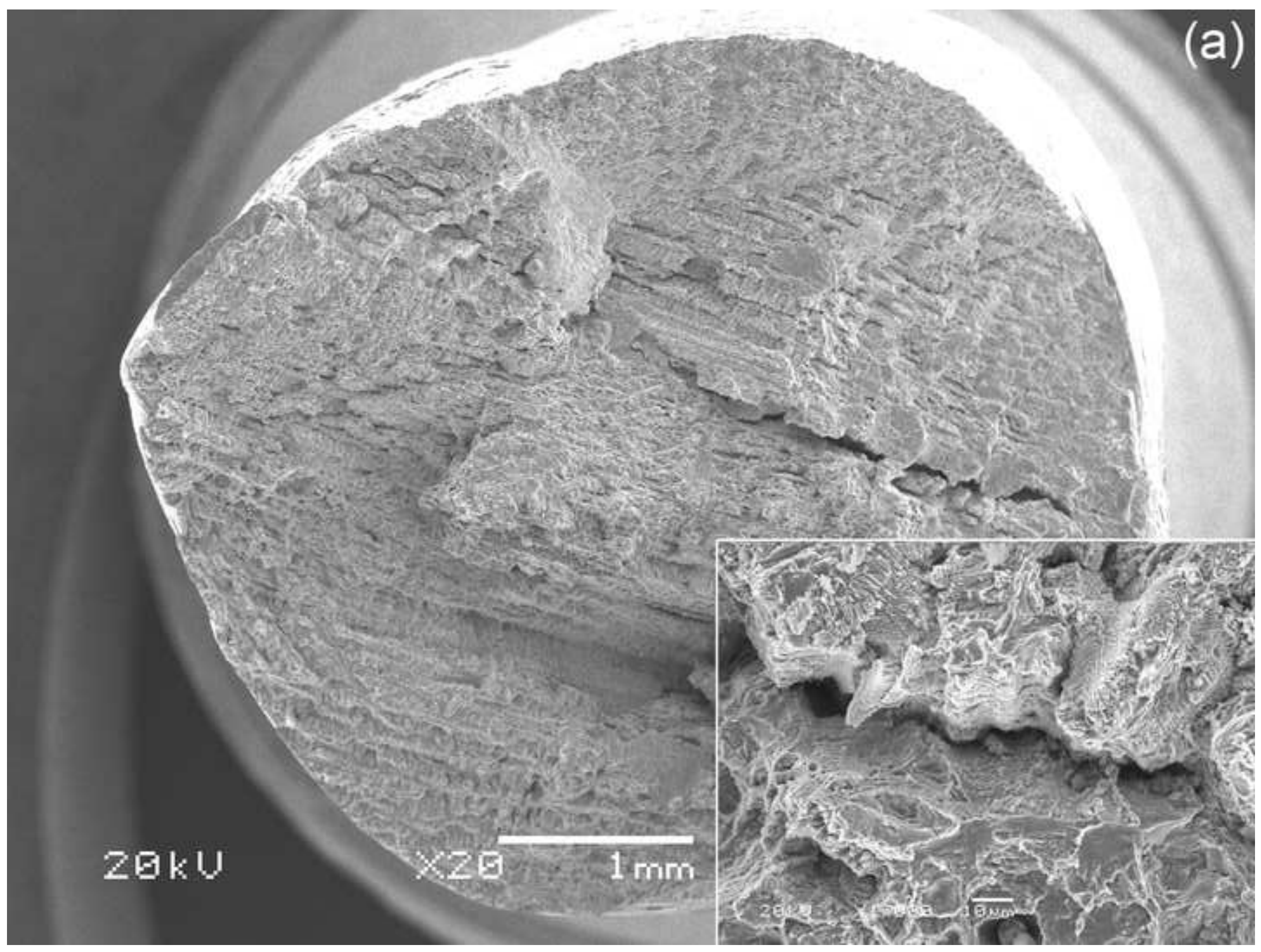



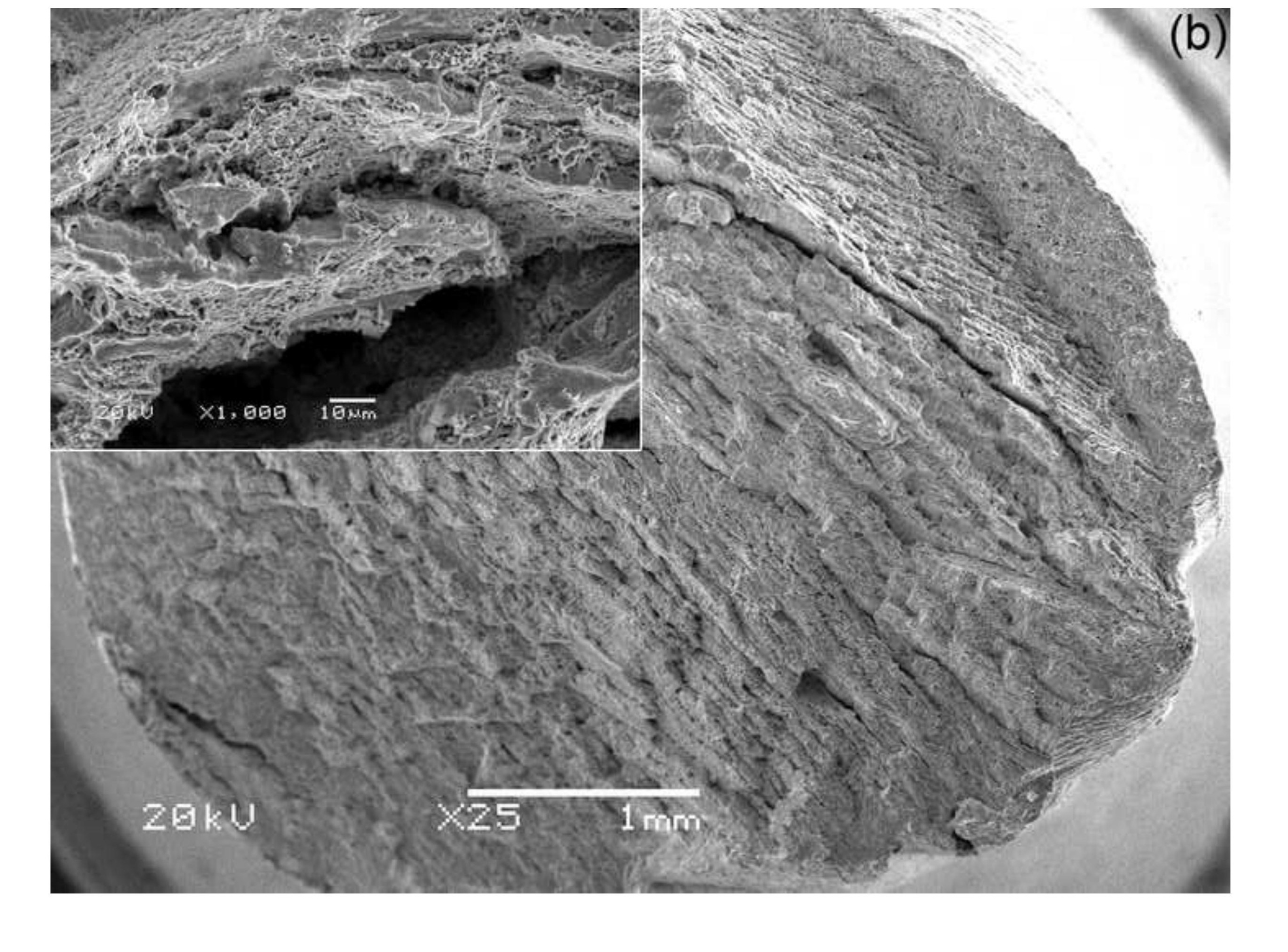

.

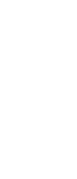



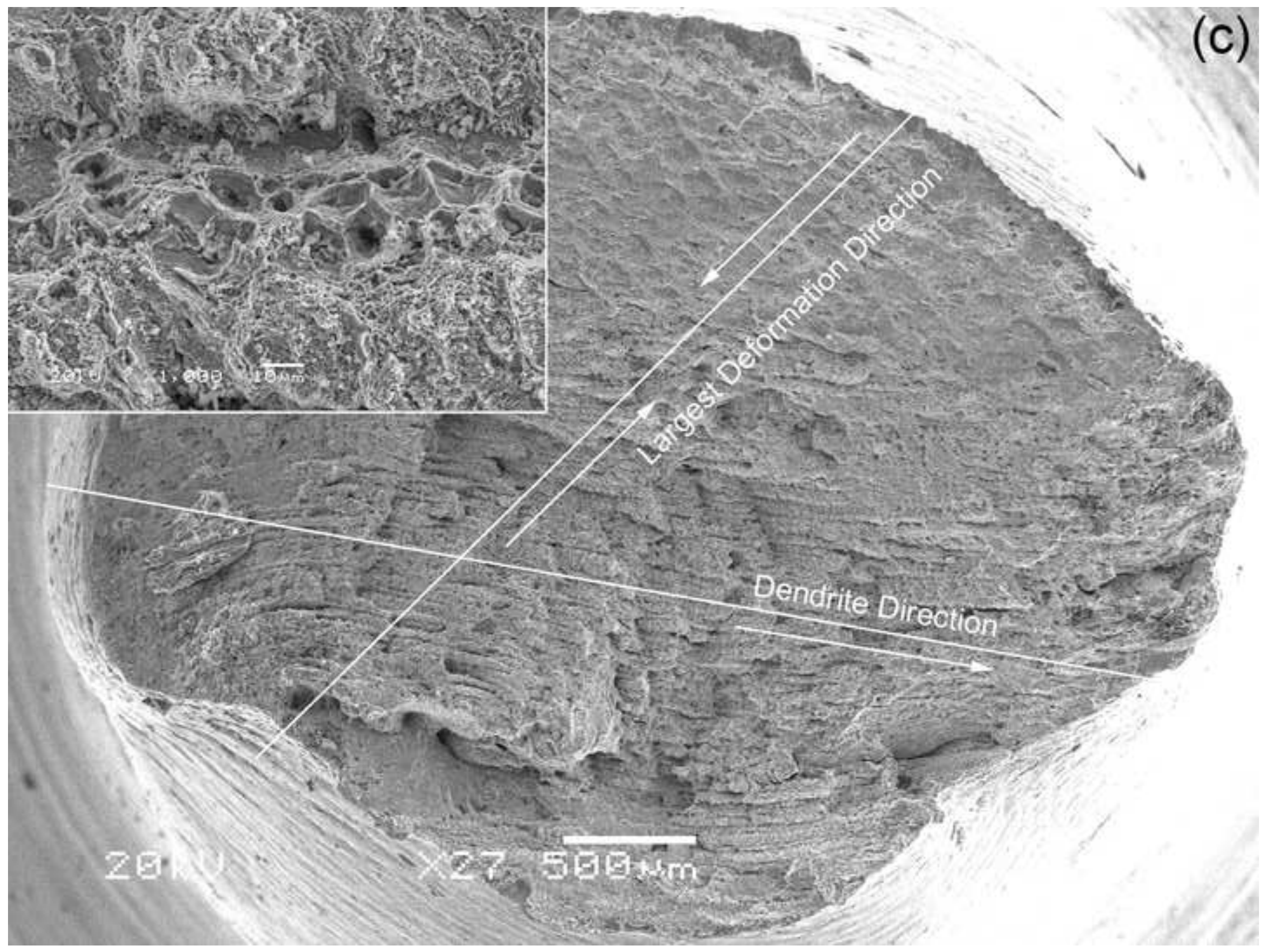


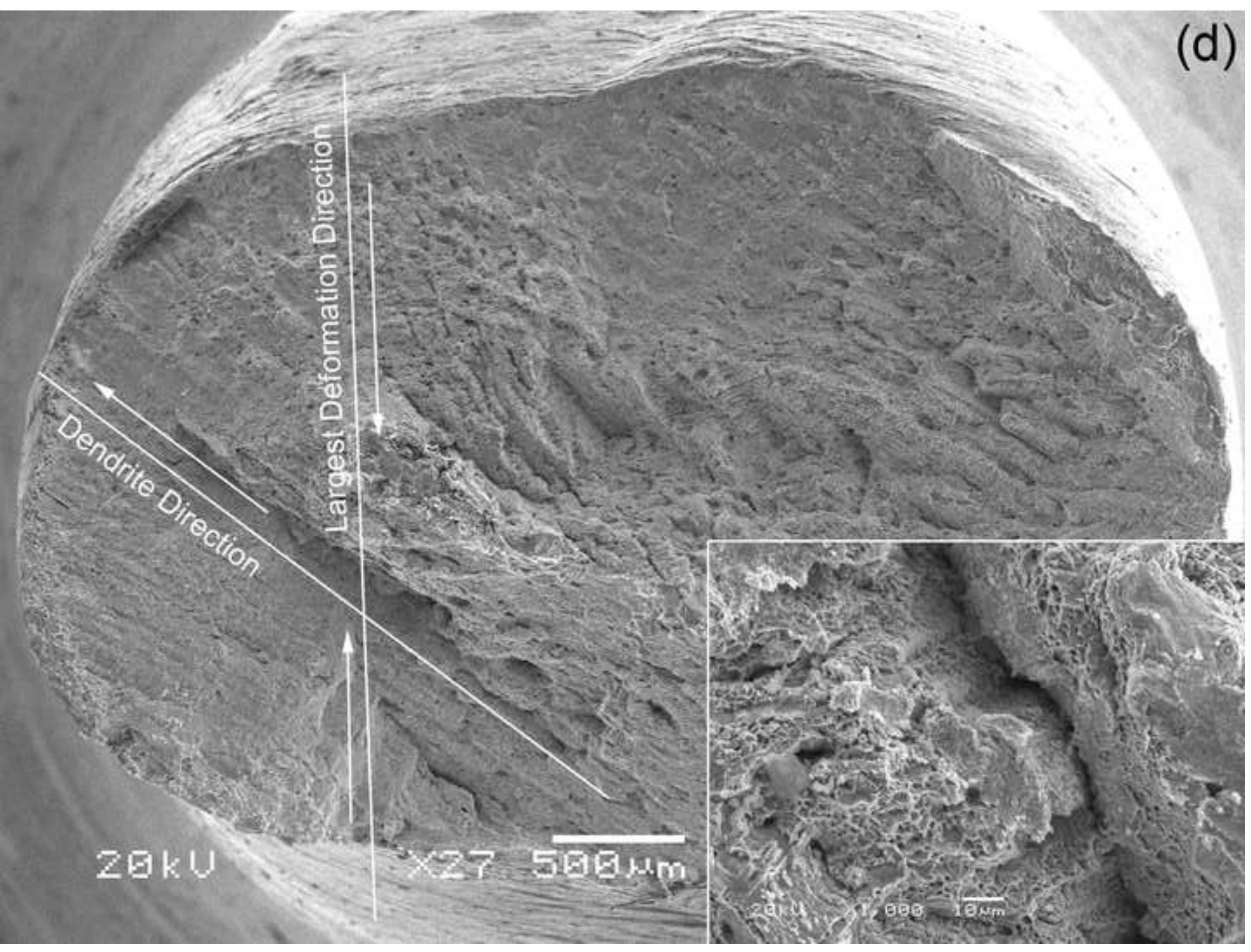

(d)

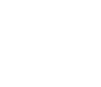

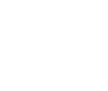

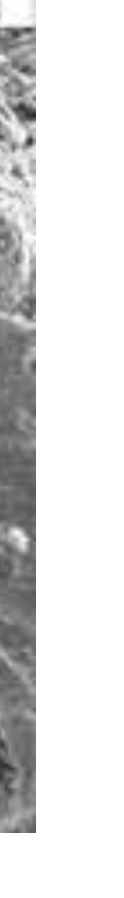
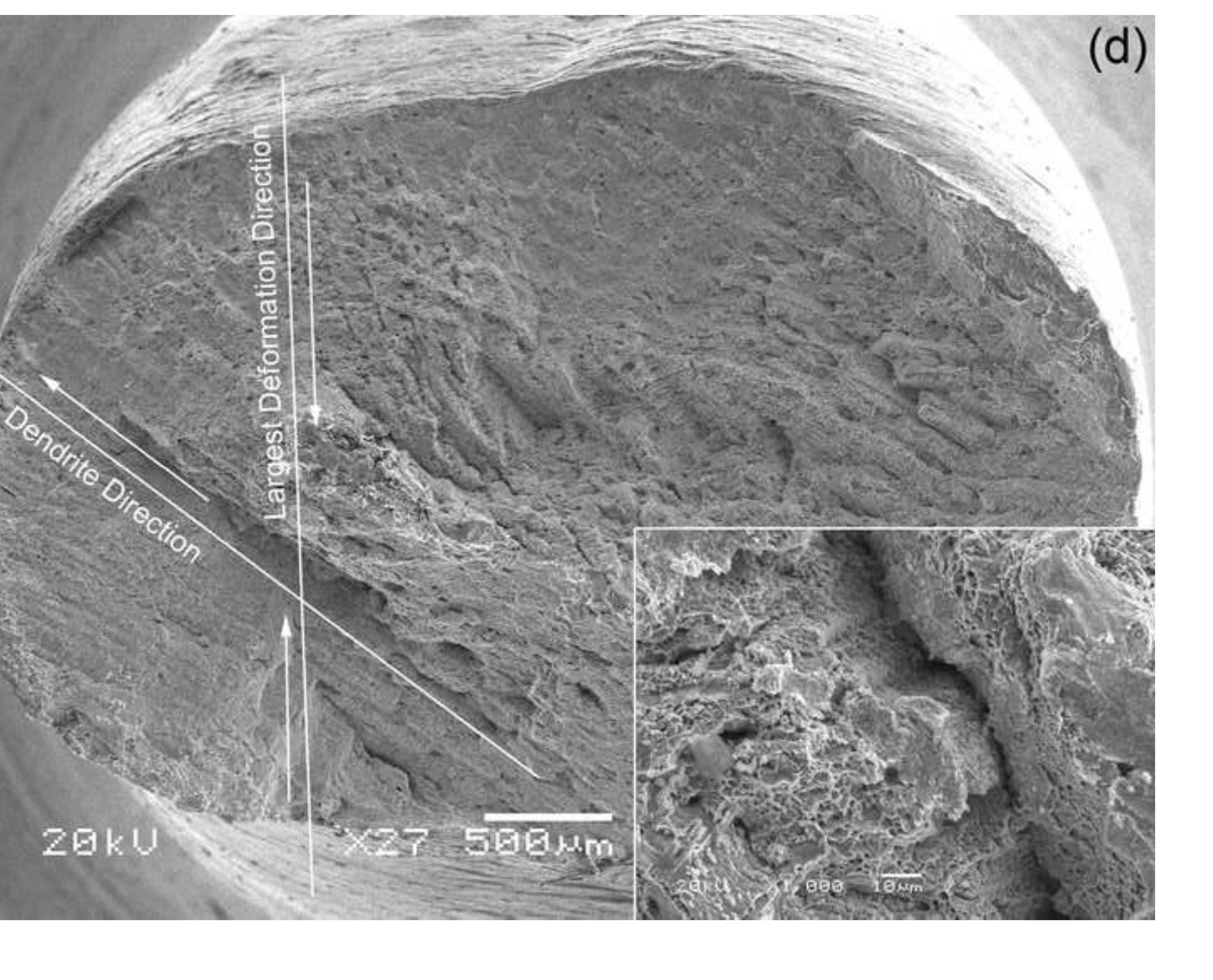


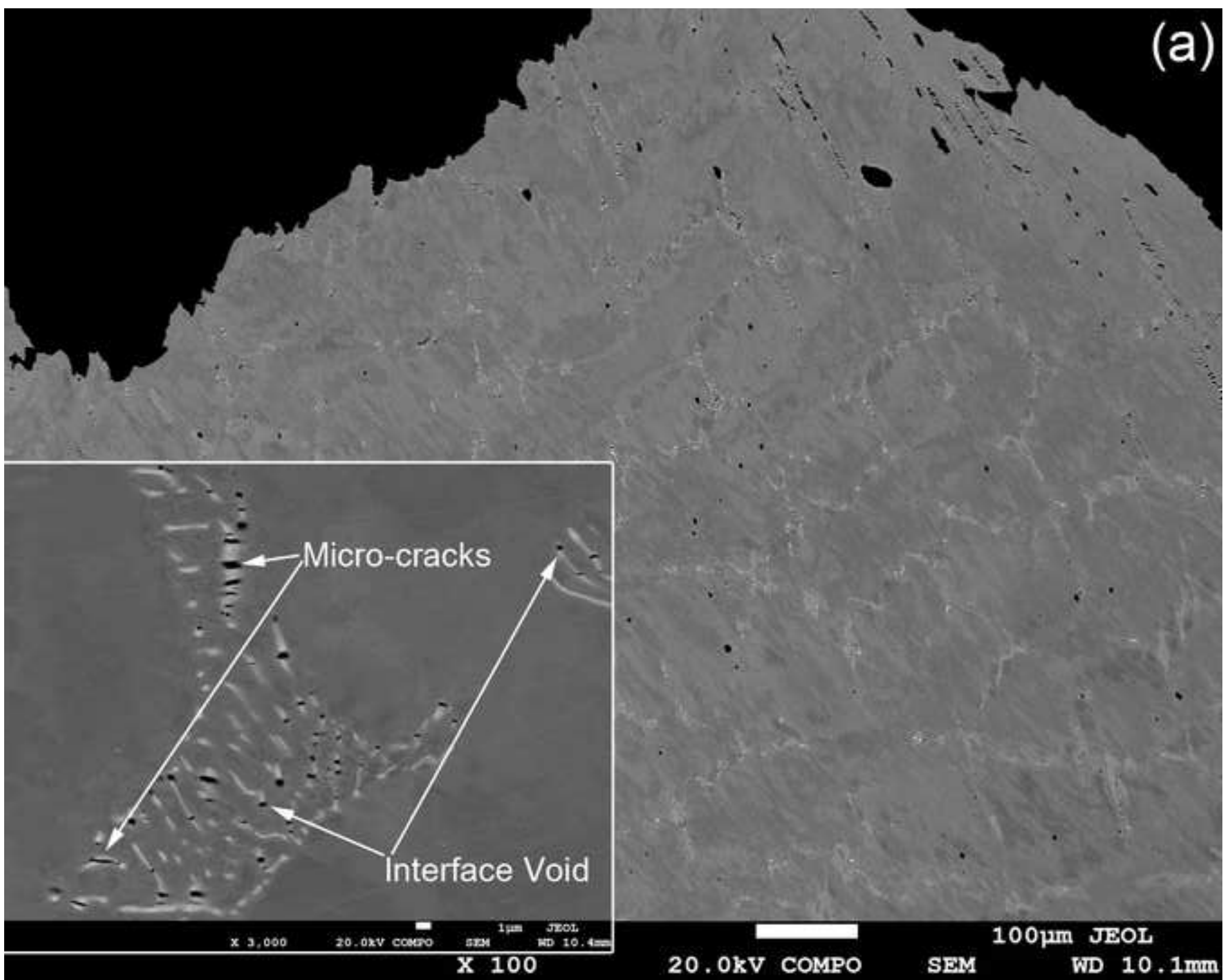

WD $10.1 \mathrm{~mm}$ 


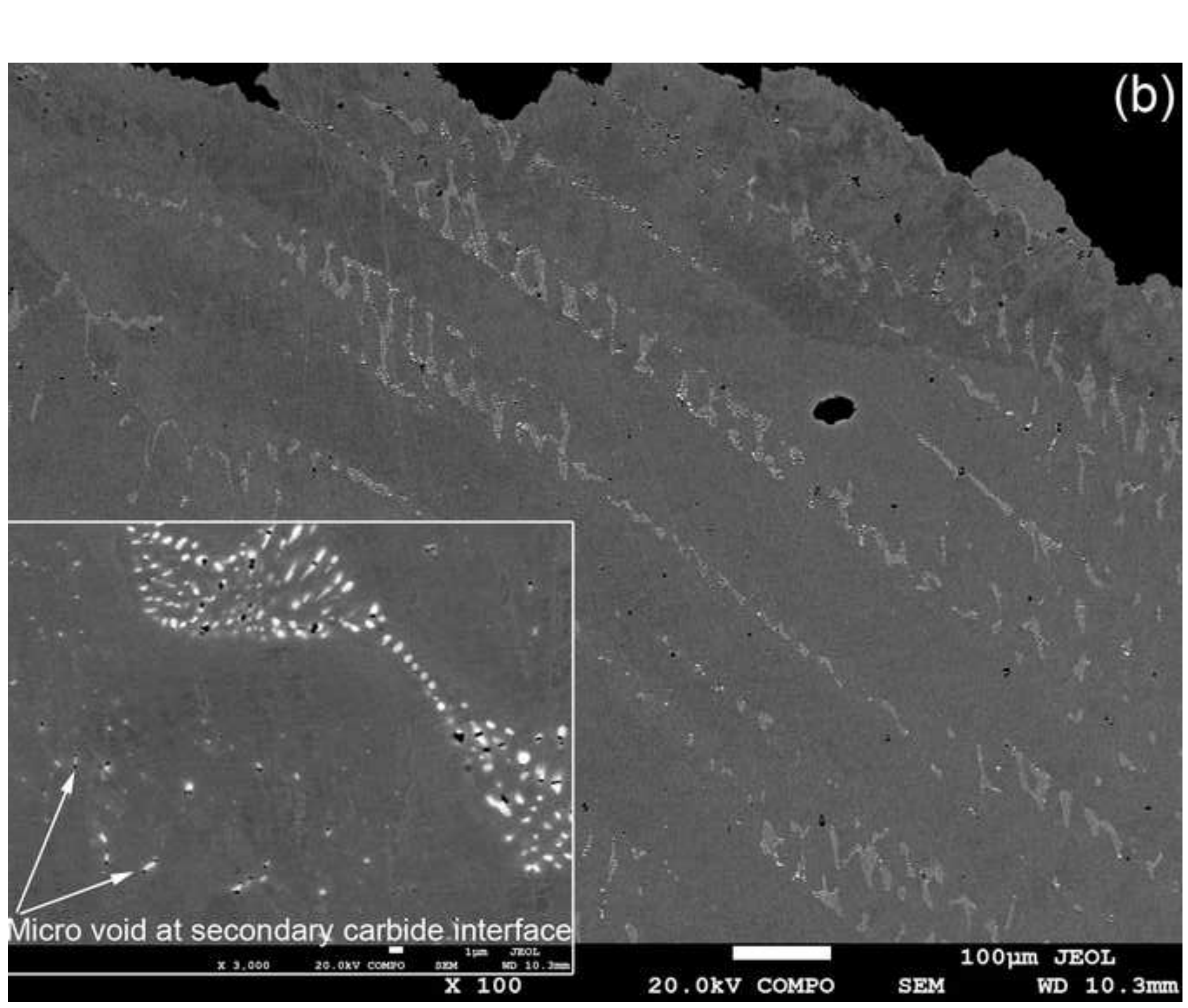




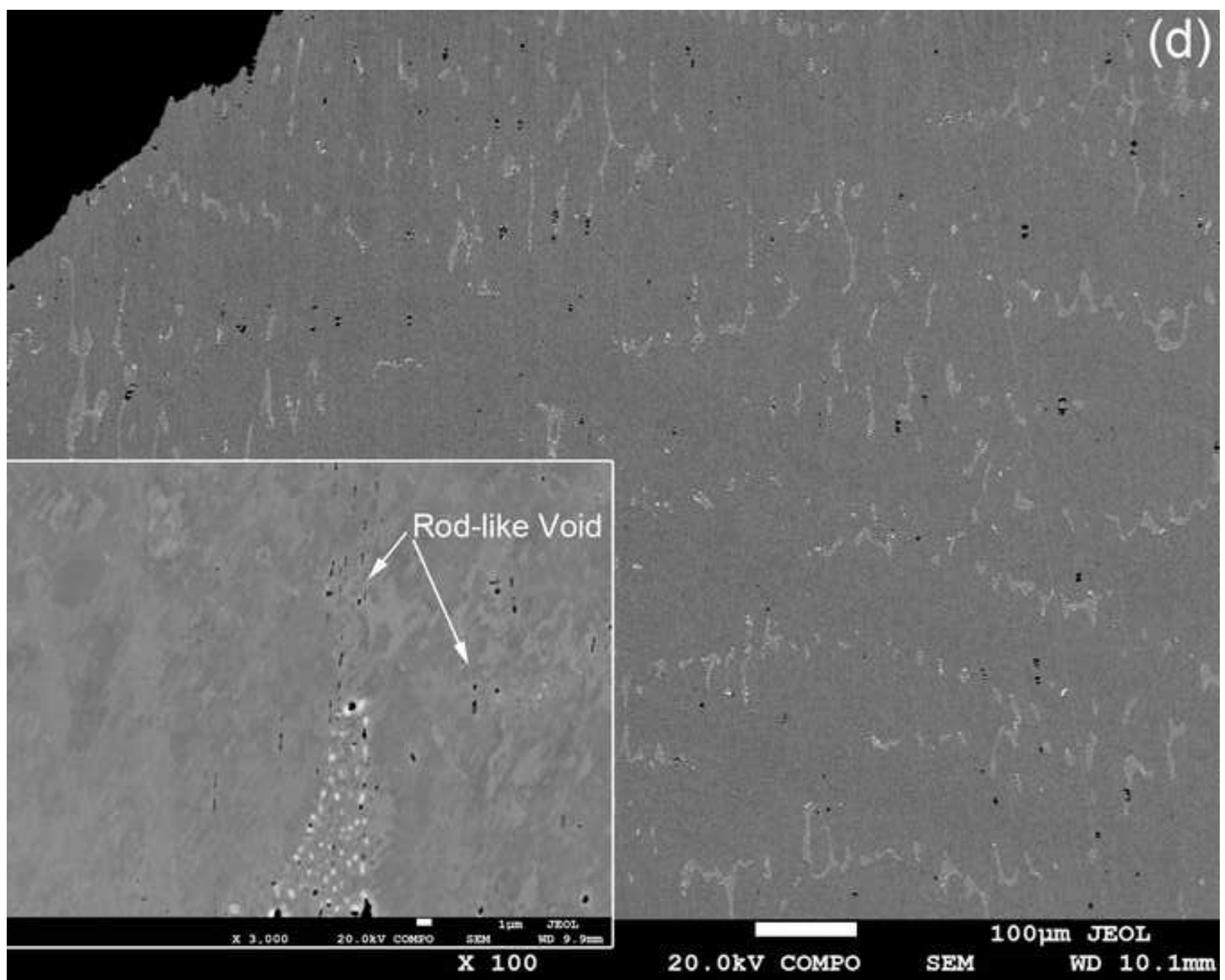




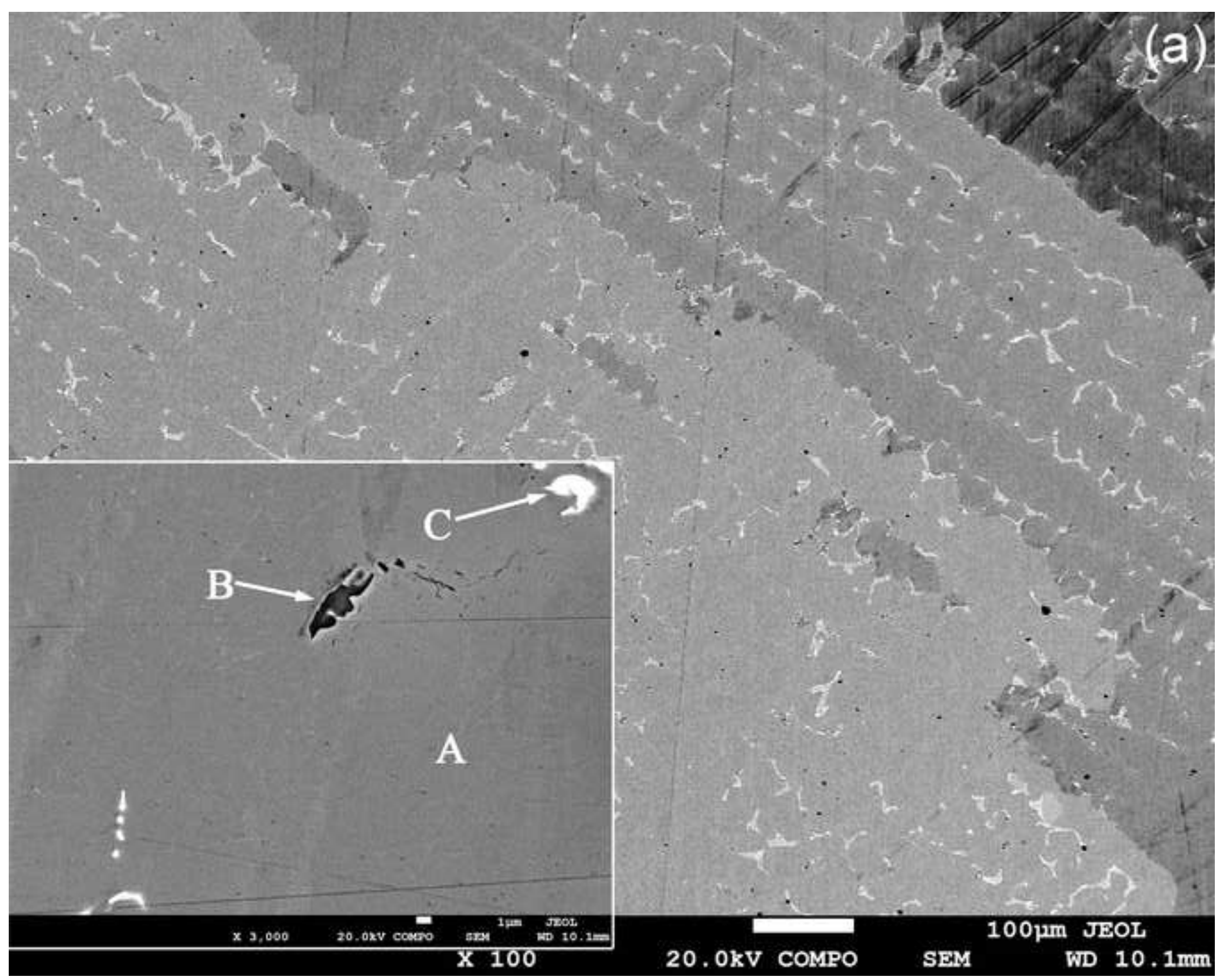




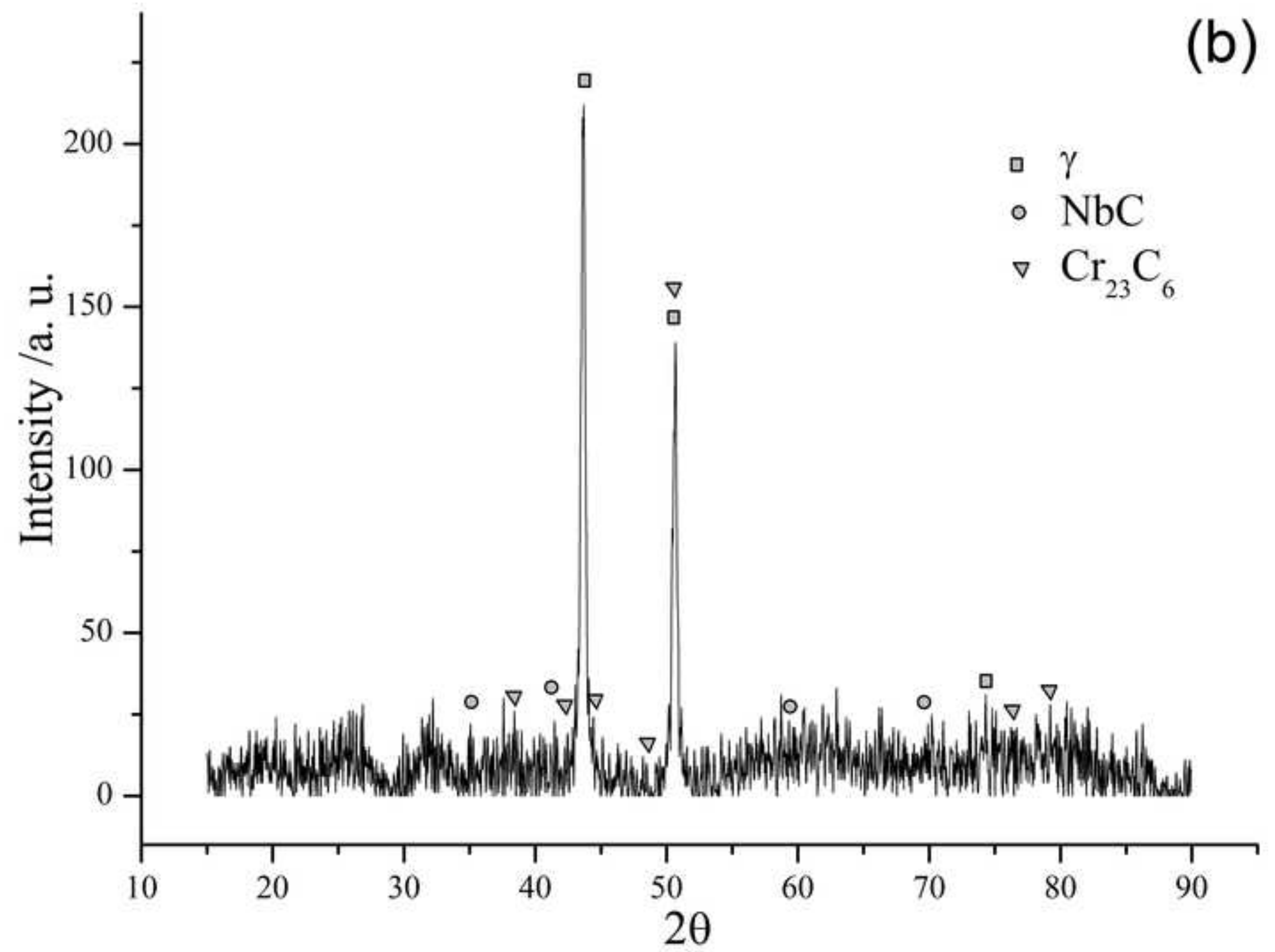



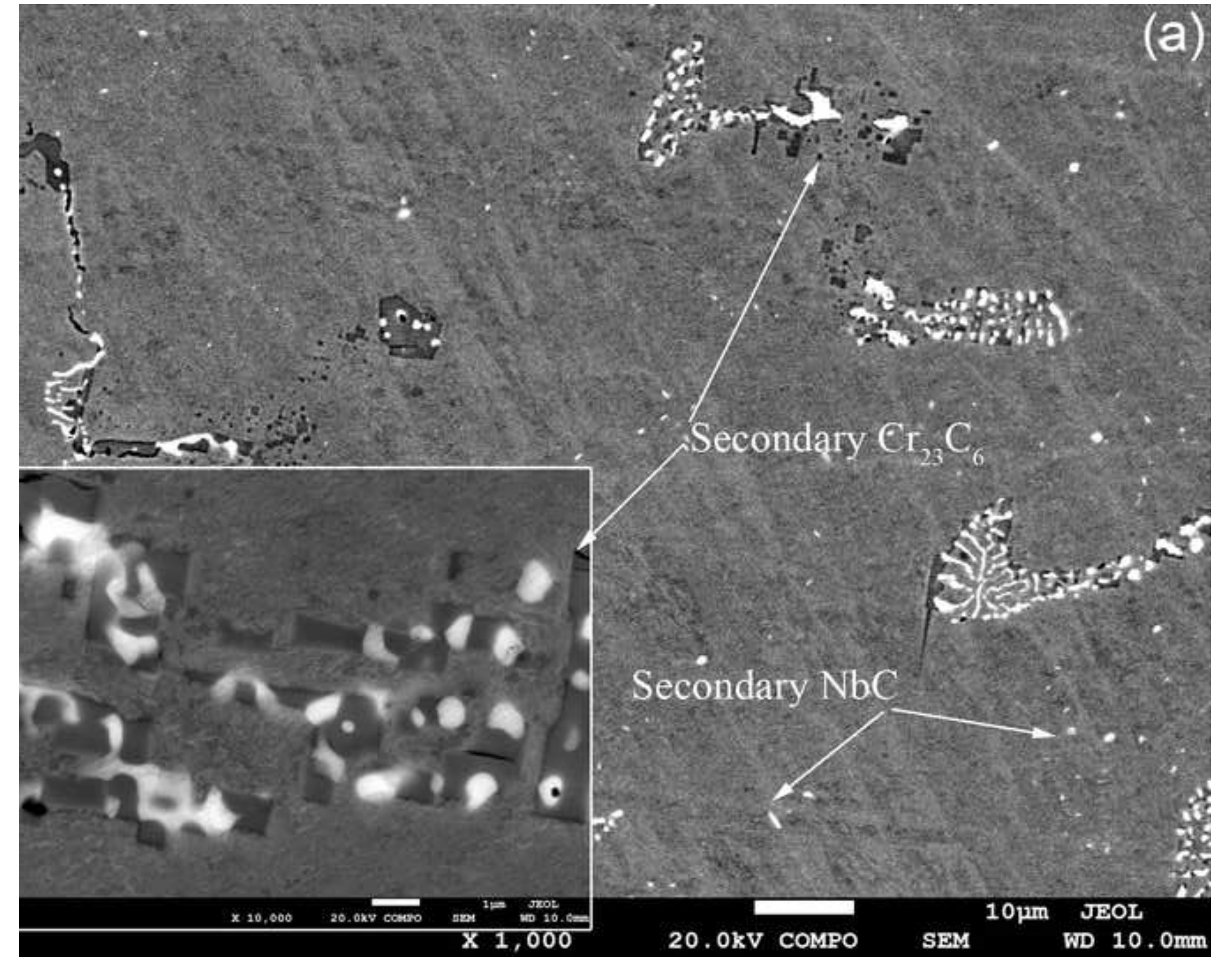

(




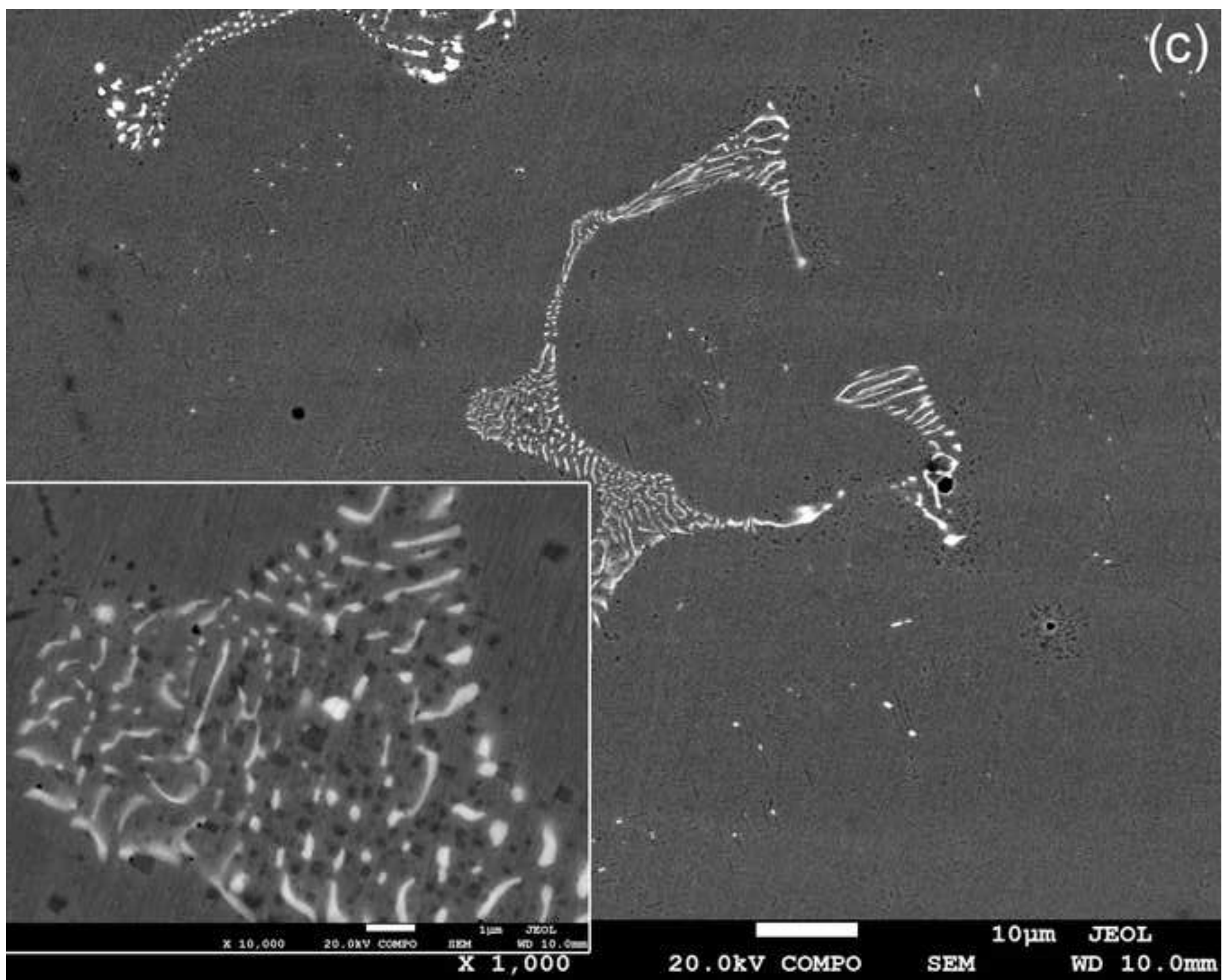




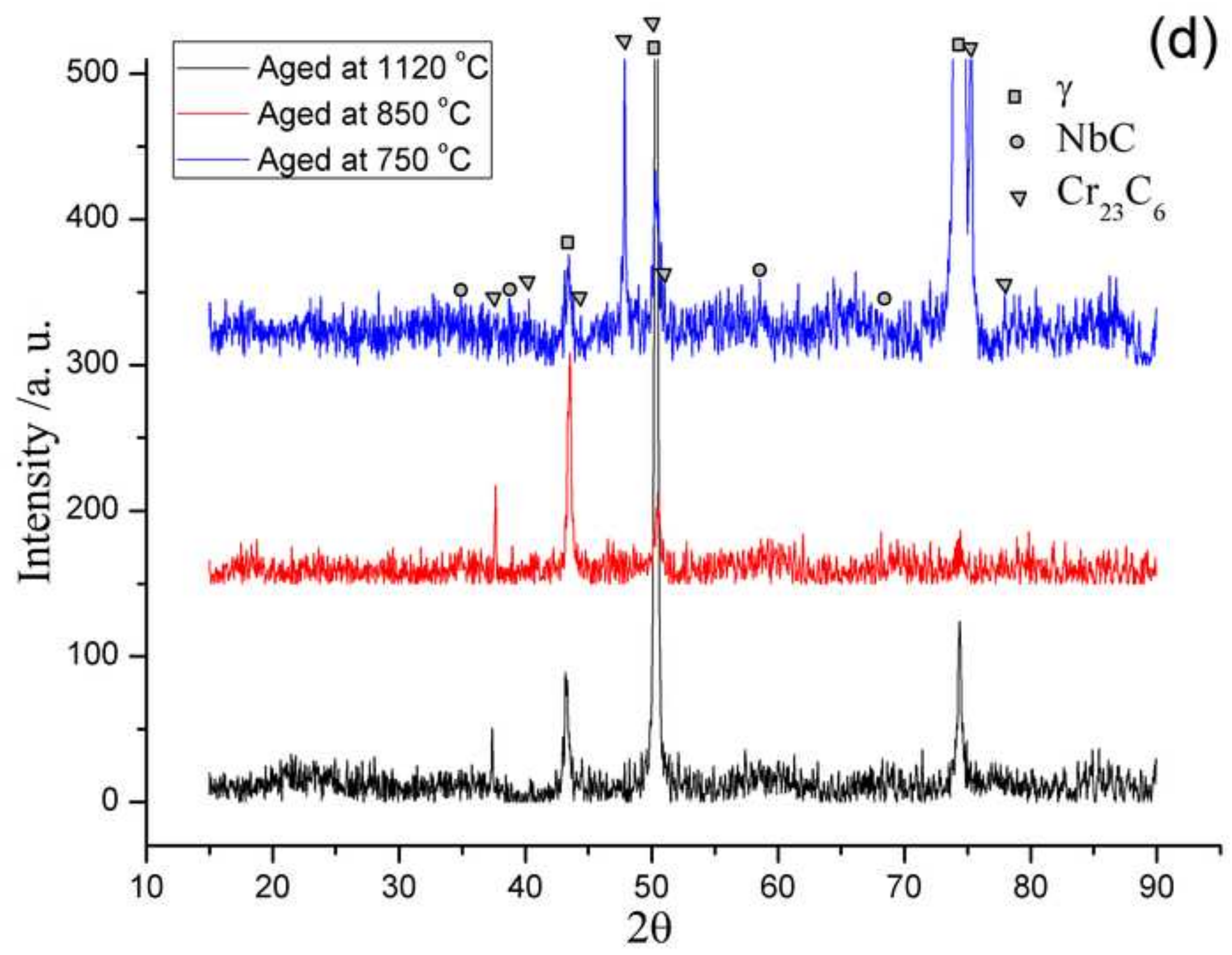



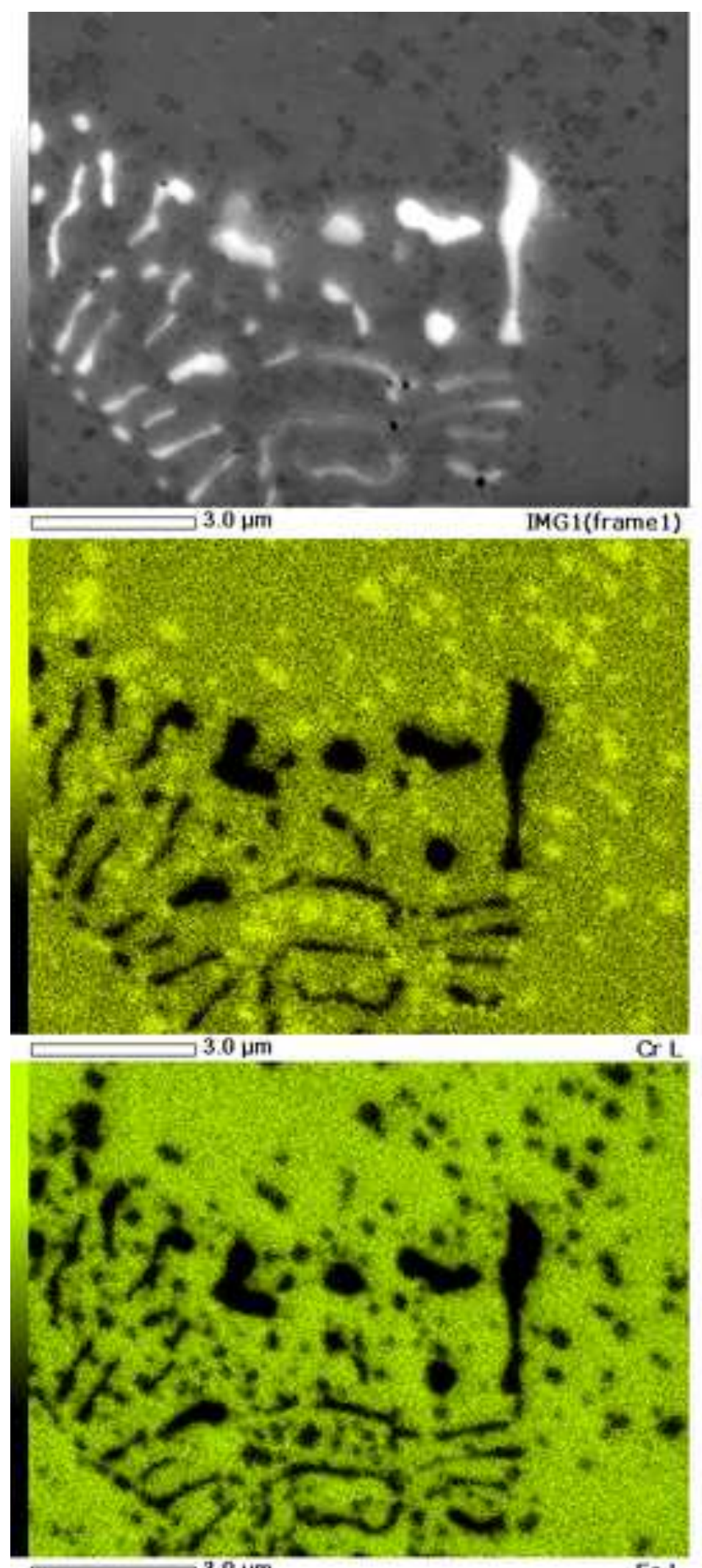

FeL $L$
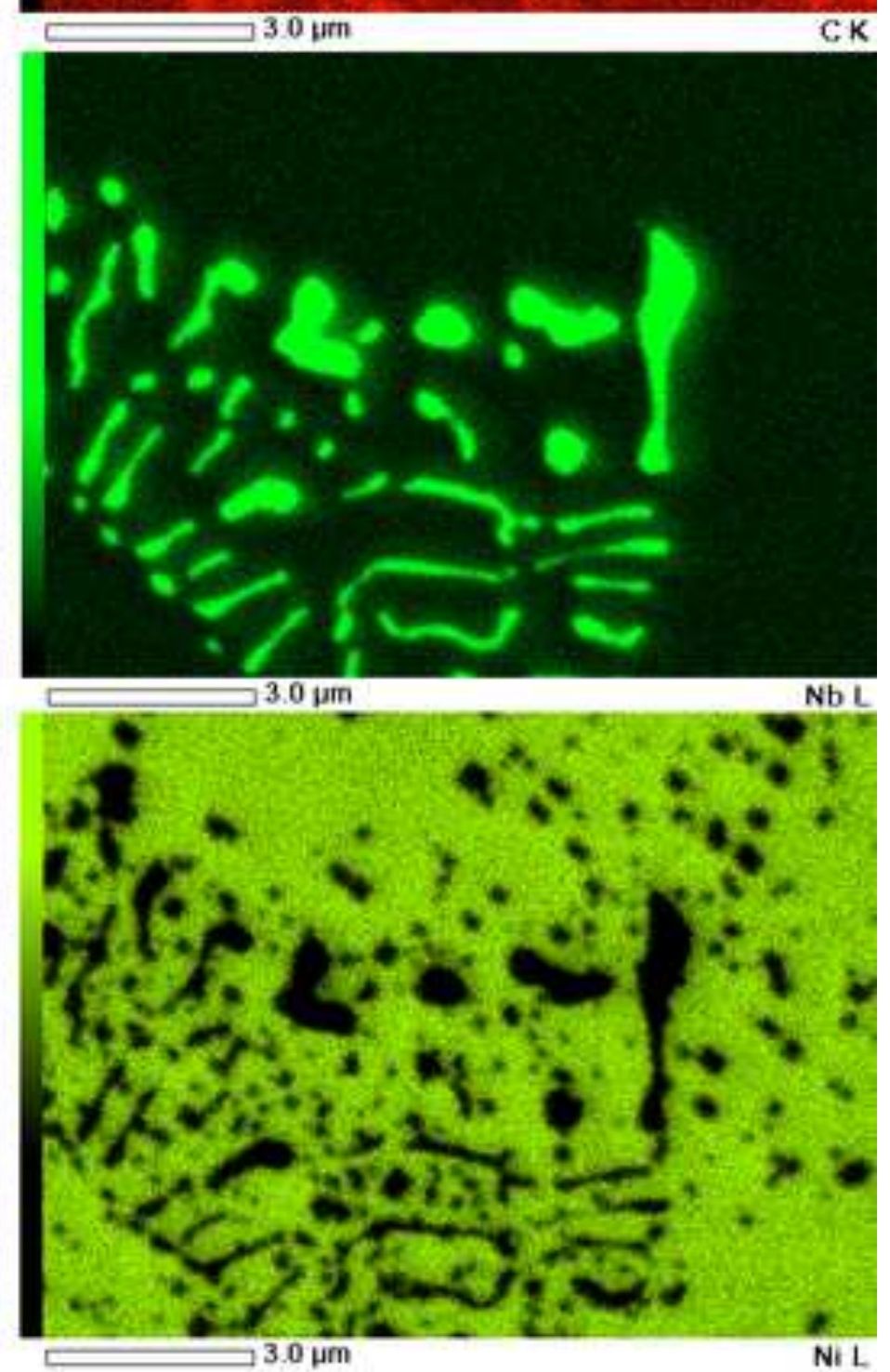
(a)

$400-1$ Cast

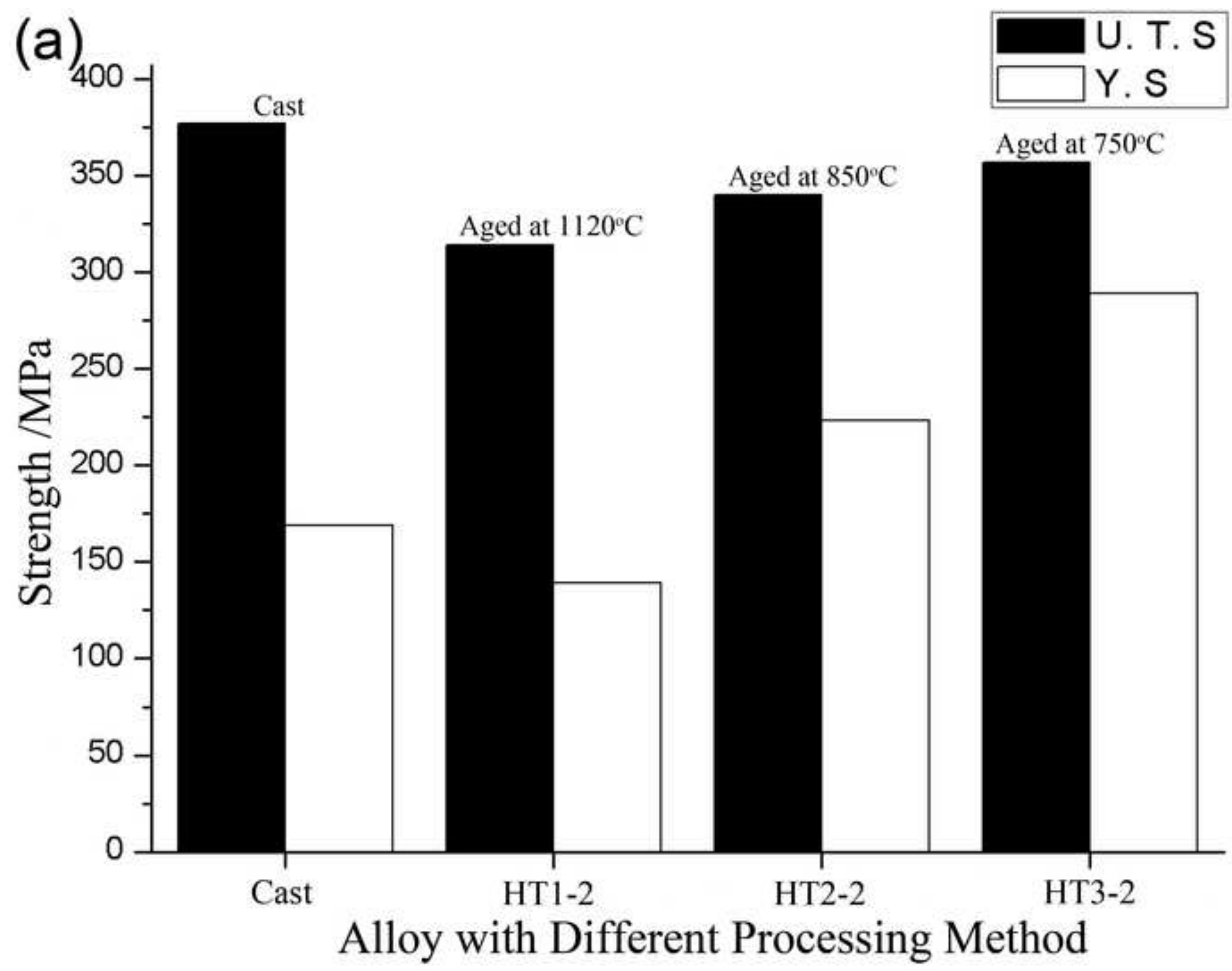




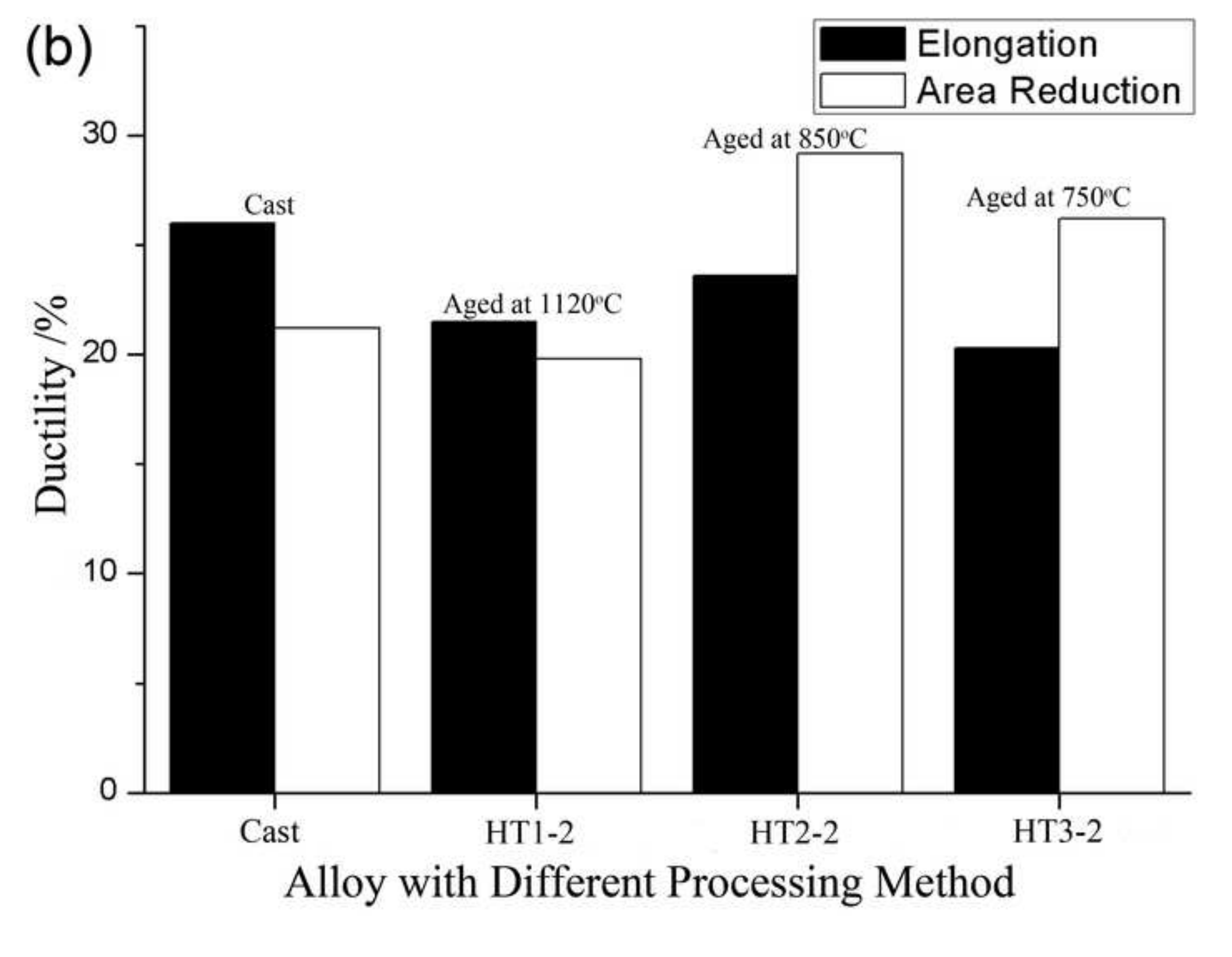




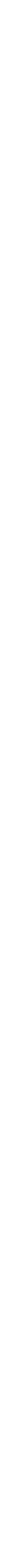

ZEkU

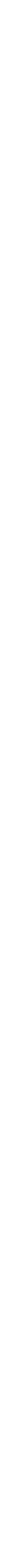

(a)
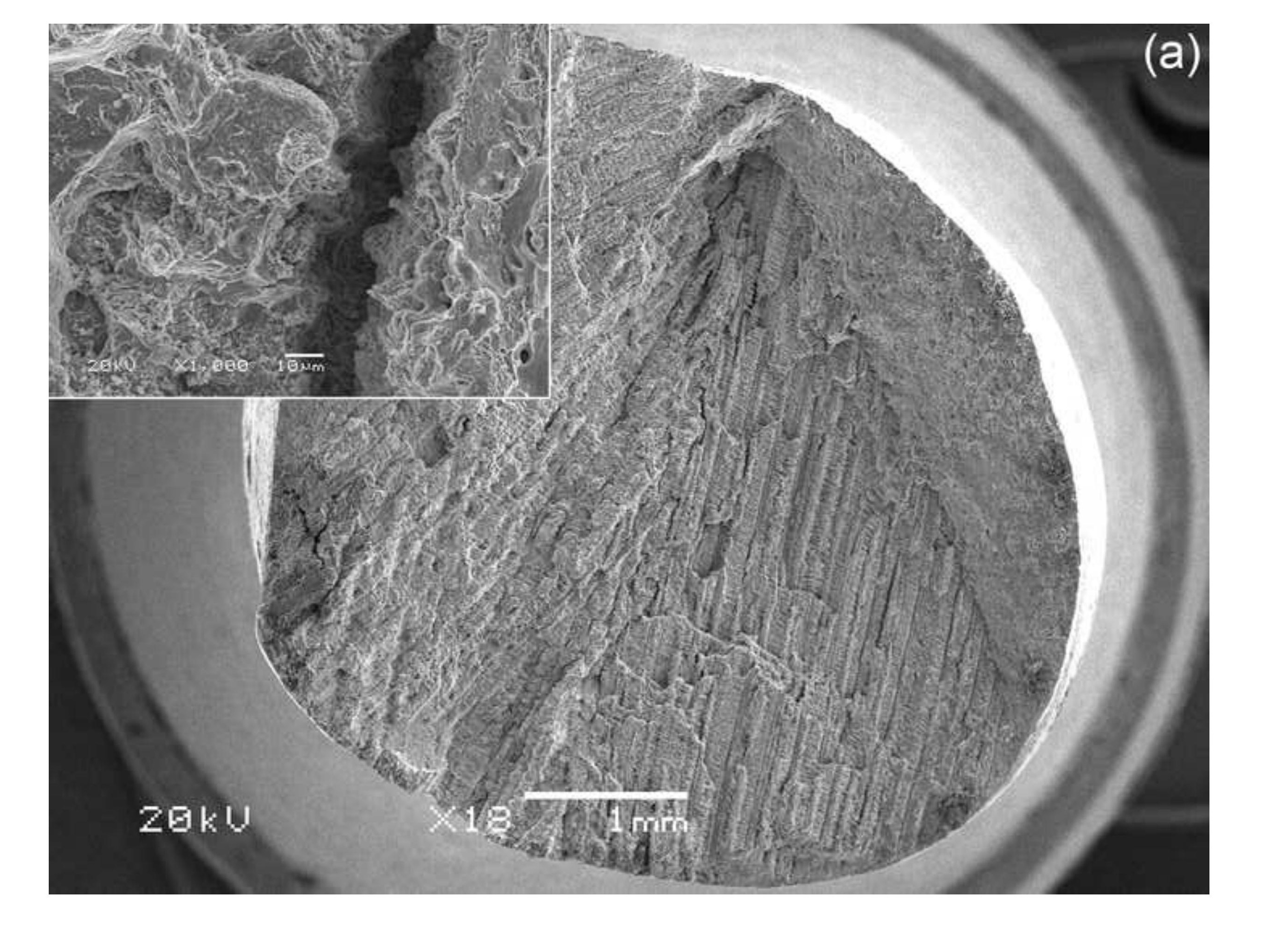

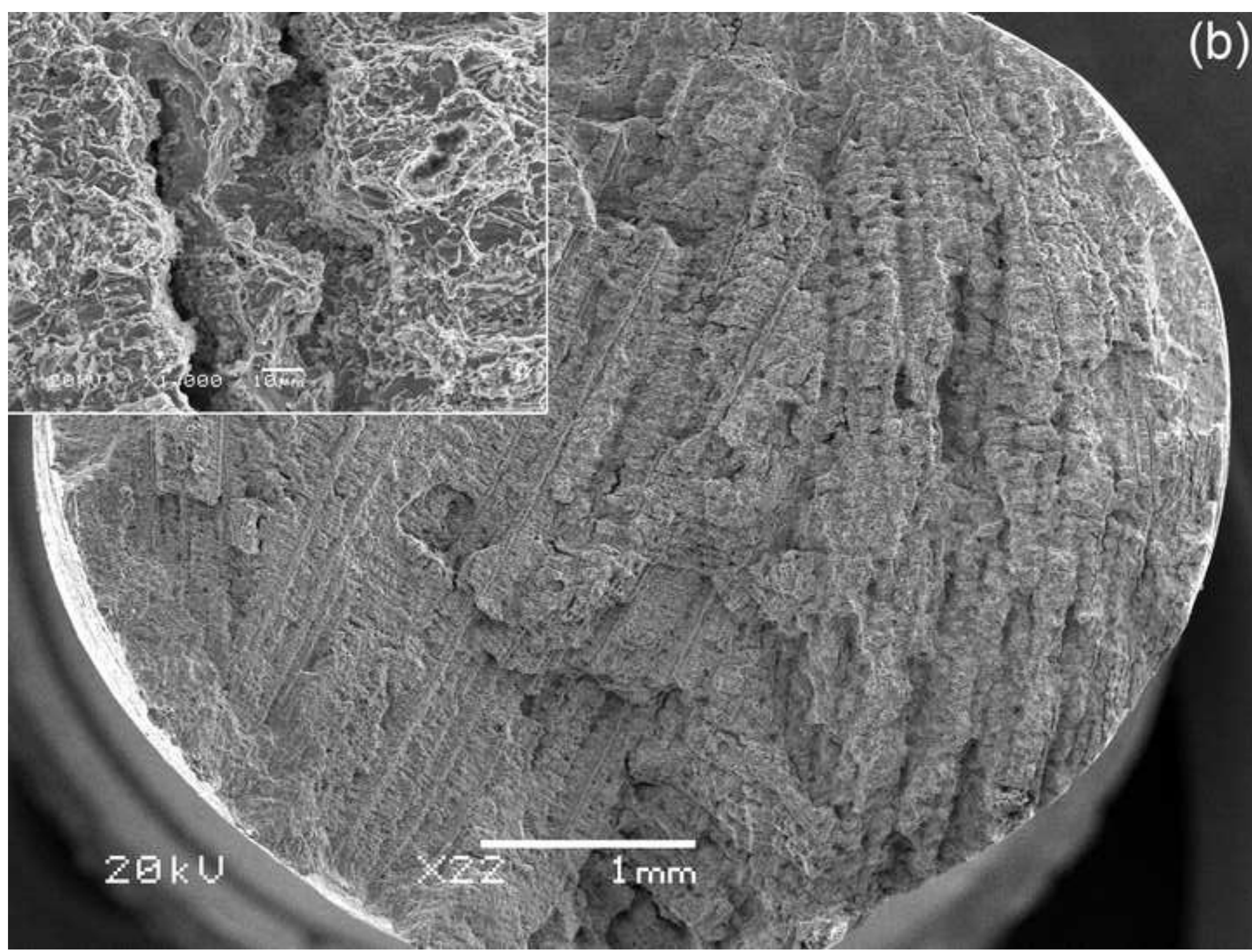

(b)

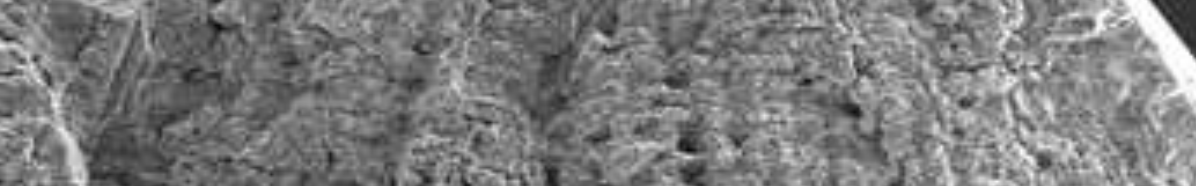

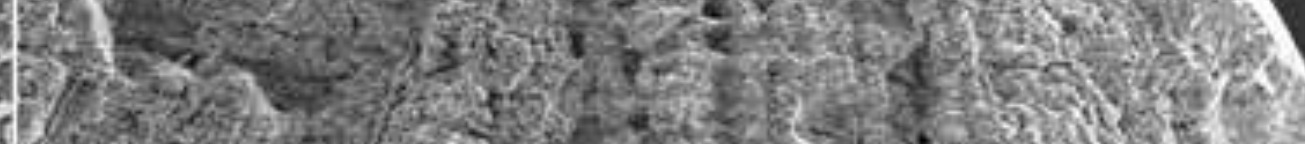
1.5.

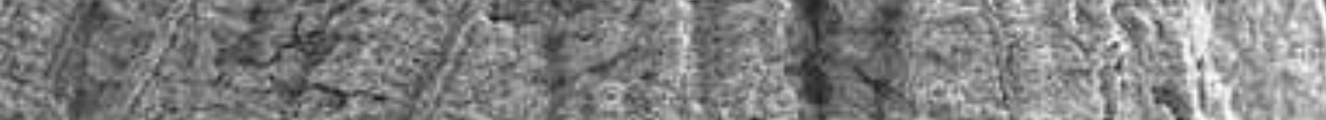

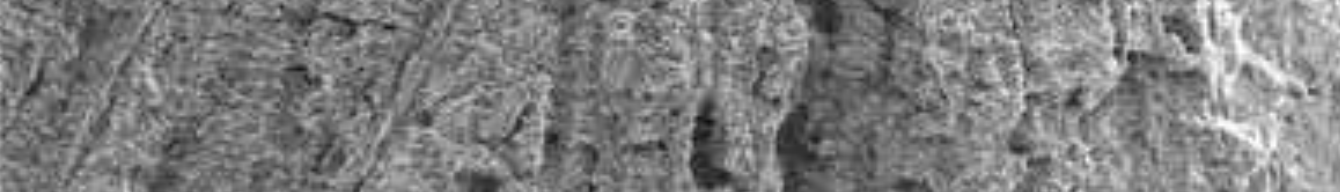

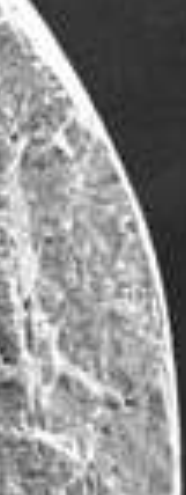
i

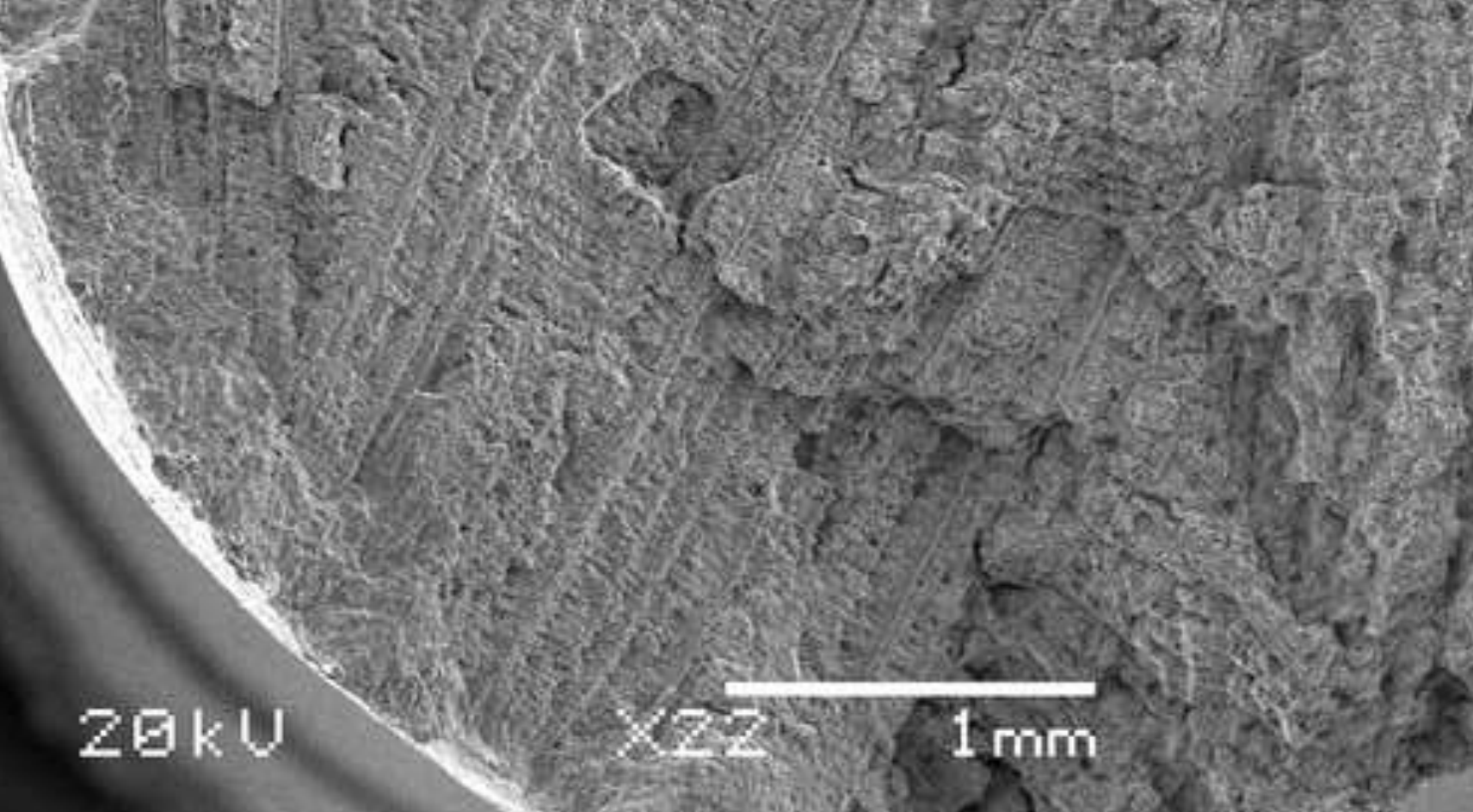




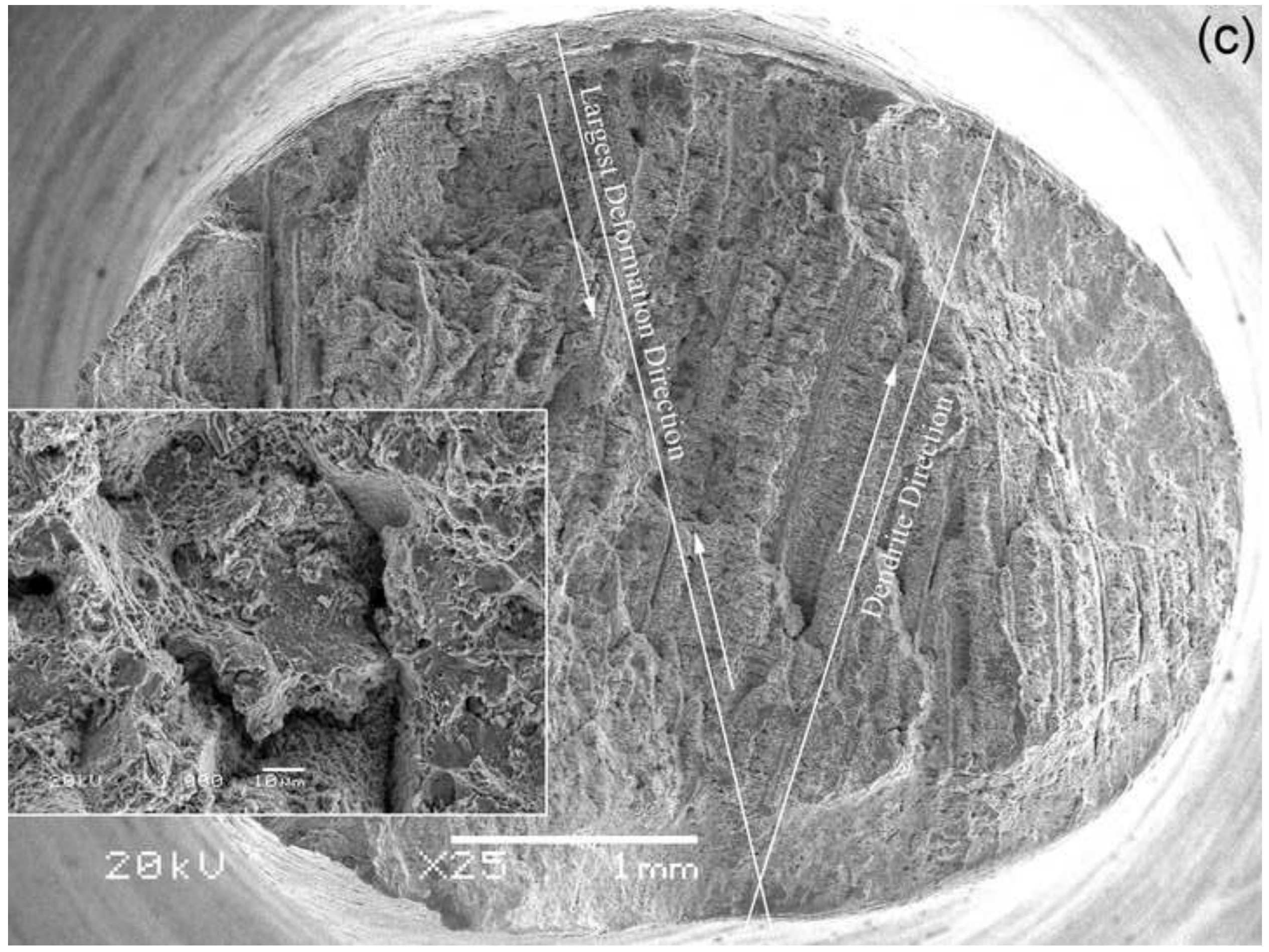




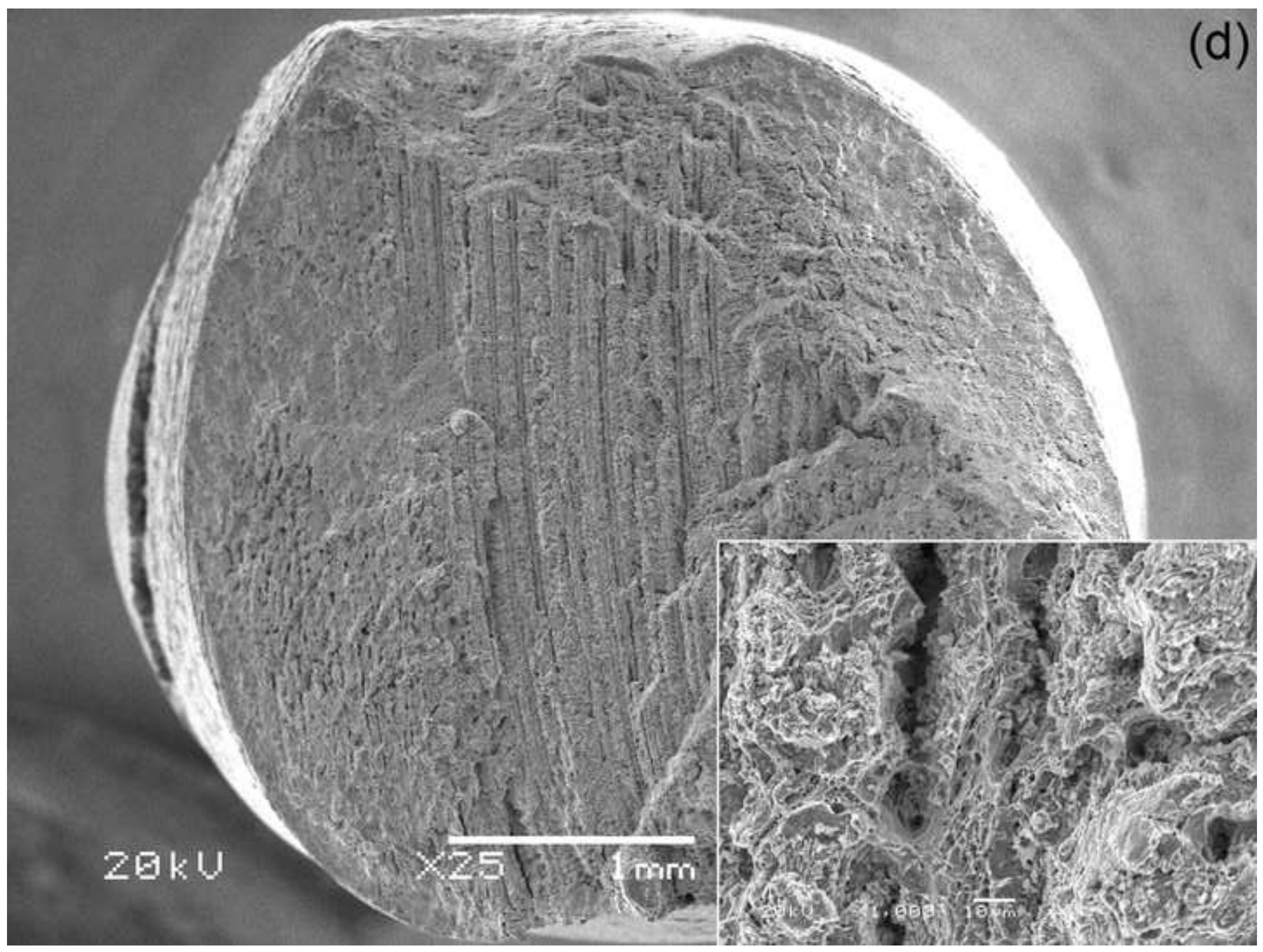




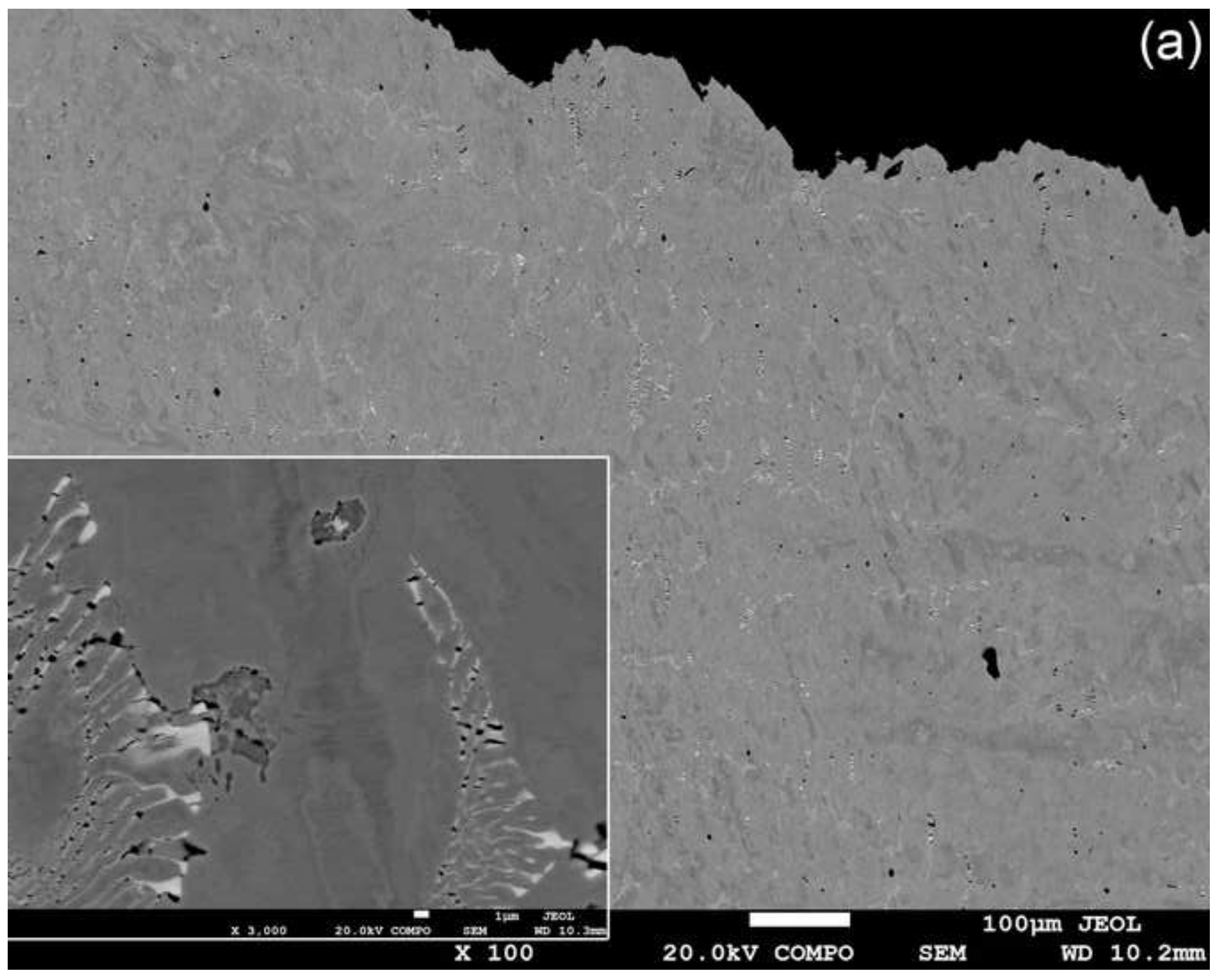




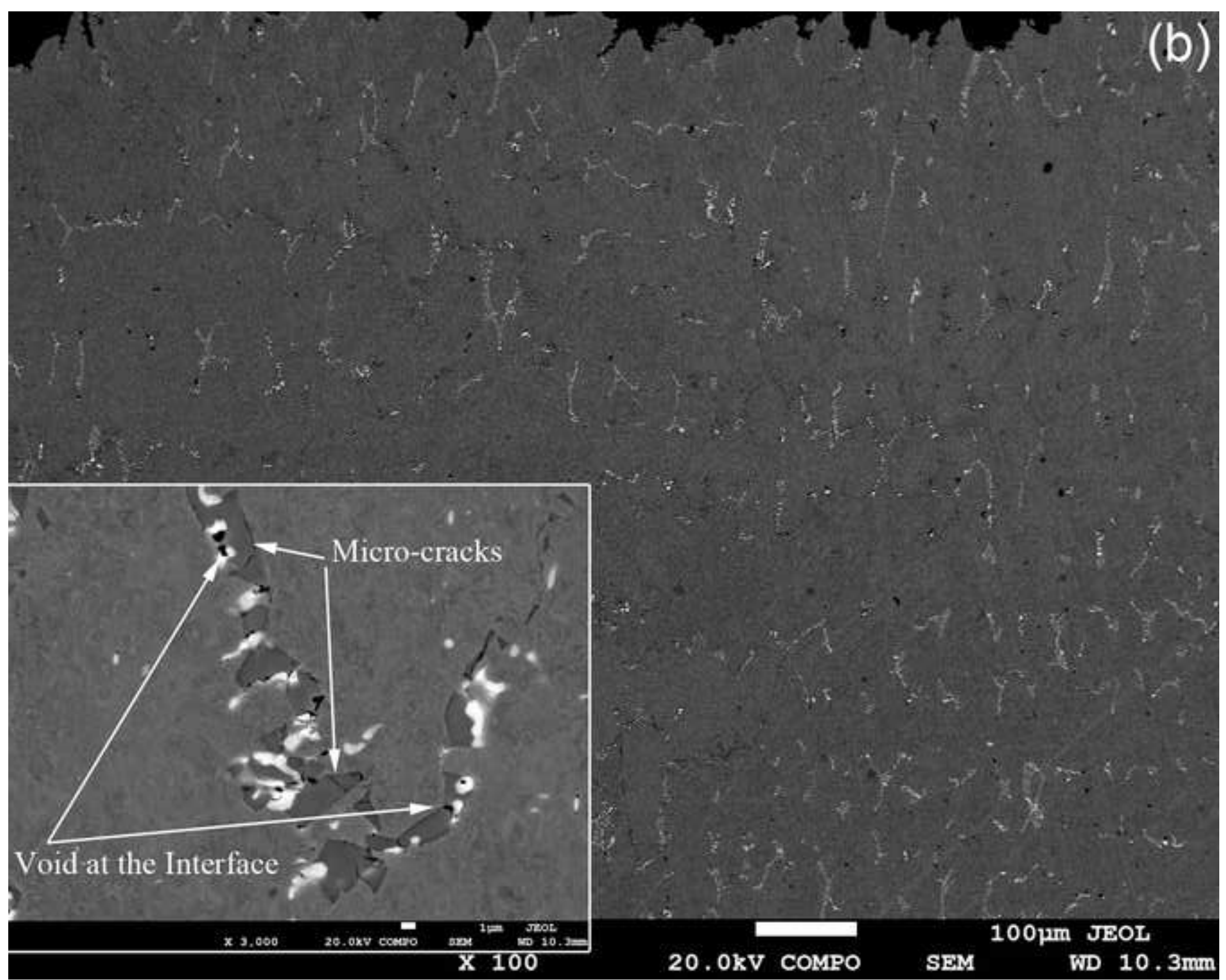




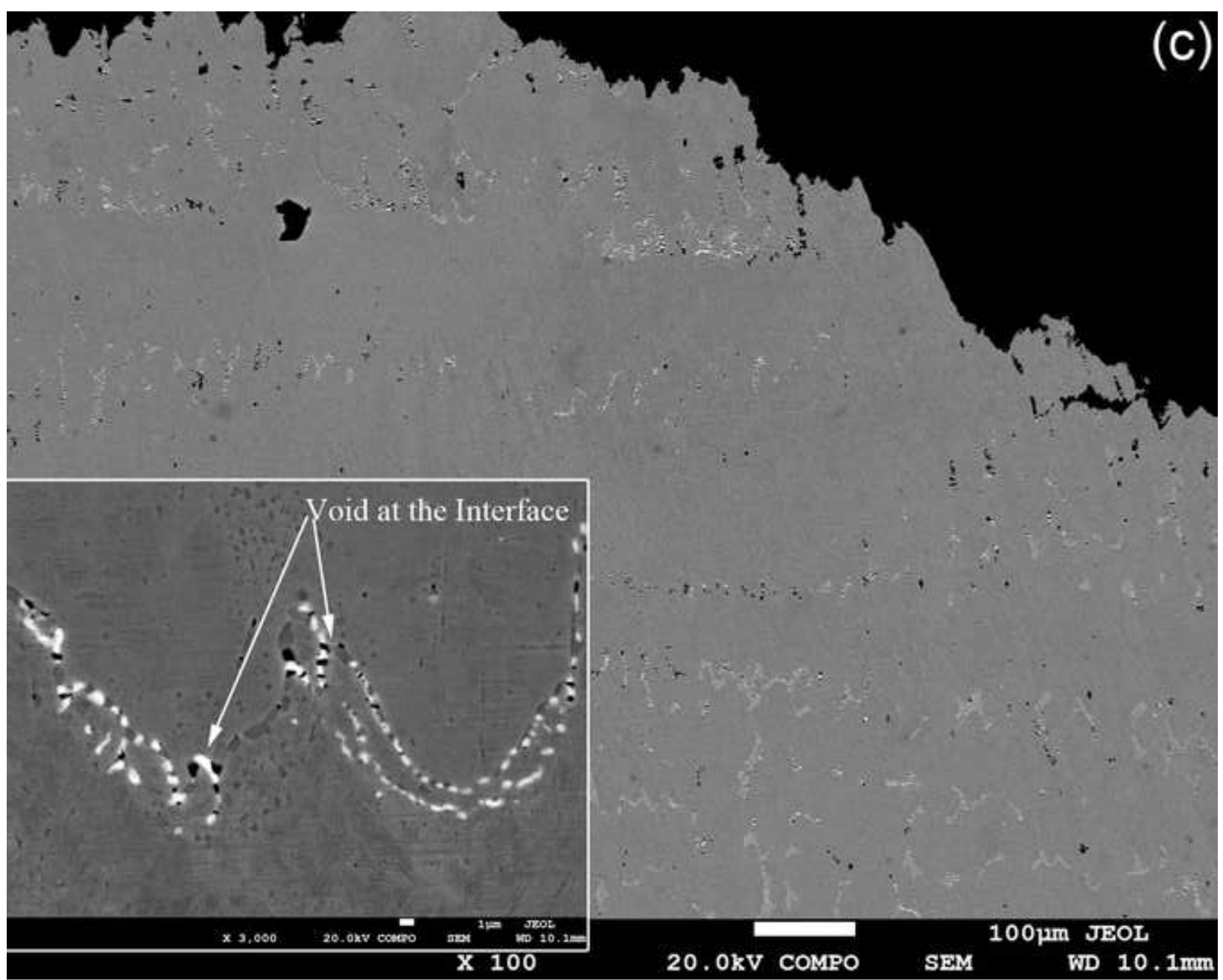




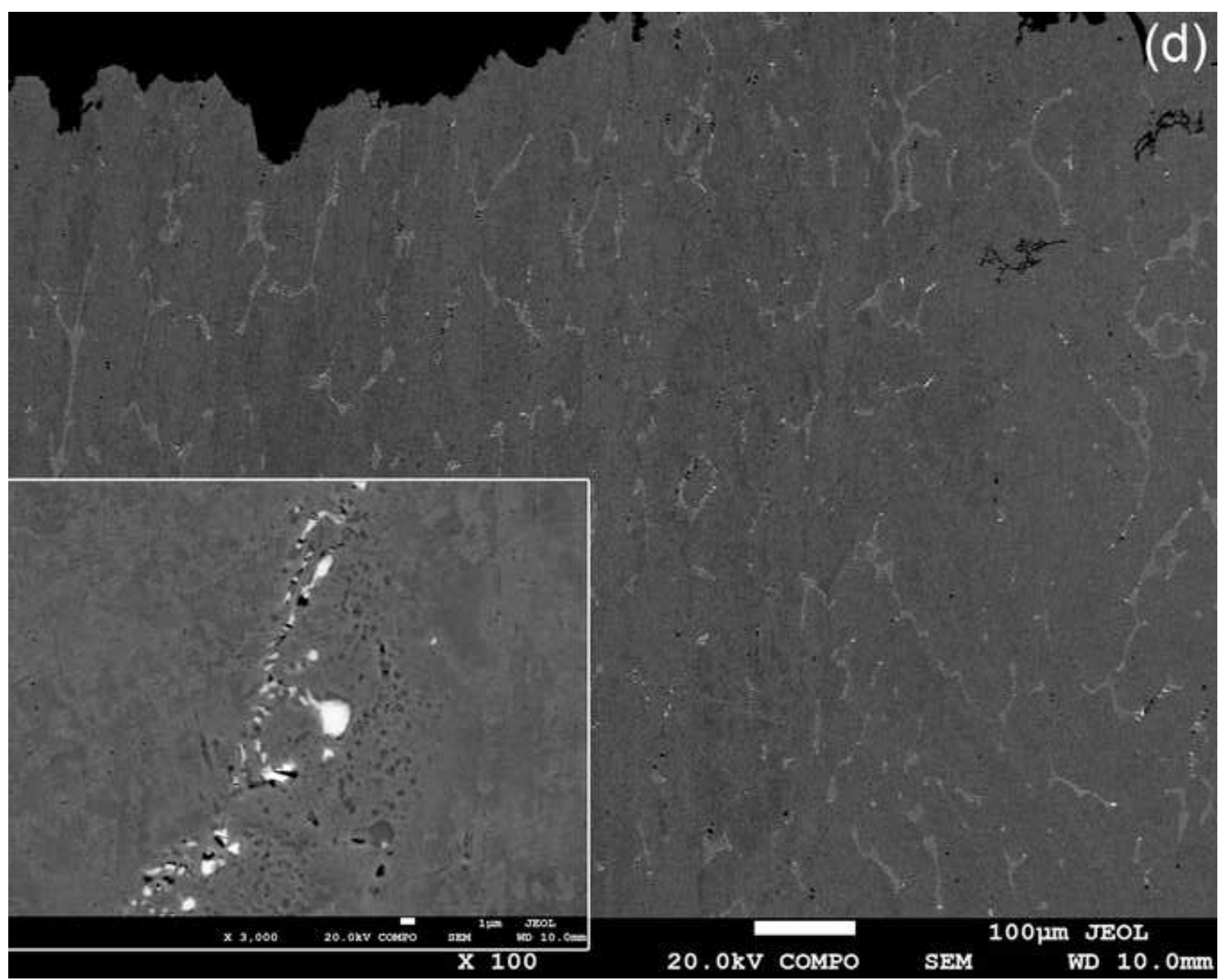

WD $10.0 \mathrm{~mm}$ 


\section{Figure Captions}

Fig. 1 Microstructure of as-cast 20Cr32Ni1Nb alloy: (a) Microstructure, (b) Phase constitution

Fig. 2 Microstructure evolution of $20 \mathrm{Cr} 32 \mathrm{Ni} 1 \mathrm{Nb}$ alloy during different aging temperature: (a) aged at $1120^{\circ} \mathrm{C}$, (b) aged at $850{ }^{\circ} \mathrm{C}$, (c) aged at $750{ }^{\circ} \mathrm{C}$, (d) phase constitution after aging

Fig. 3 EDX map analyzing result of $20 \mathrm{Cr} 32 \mathrm{Ni} 1 \mathrm{Nb}$ alloy after aging at $1120^{\circ} \mathrm{C}$ for 2 hours

Fig. 4 Morphology and its precipitation orientation of the secondary (a) $\mathrm{Cr}_{23} \mathrm{C}_{6}$ and (b) $\mathrm{NbC}$ in the HT2-1 and HT1-1 alloy, respectively.

Fig. 5 The $750{ }^{\circ} \mathrm{C}$ tensile test results of the 20Cr32Ni1Nb alloy: (a) Strength, (b) Ductility (The data of CT 15C alloy refer to the ASTM: A351 Grade CT15C)

Fig. 6 Morphology of the alloy fracture surface: (a) cast alloy, (b) $1120{ }^{\circ} \mathrm{C}$ aged alloy, (c) $850{ }^{\circ} \mathrm{C}$ aged alloy, (d) $750{ }^{\circ} \mathrm{C}$ aged alloy

Fig. 7 Cross-section microstructure of the fracture subsurface: (a) cast alloy, (b) $1120{ }^{\circ} \mathrm{C}$ aged alloy, (c) $850{ }^{\circ} \mathrm{C}$ aged alloy, (d) $750{ }^{\circ} \mathrm{C}$ aged alloy

Fig. 8 Microstructure of as-cast high C-adopted 20Cr32Ni1Nb alloy: (a) Microstructure, (b) Phase constitution

Fig. 9 Microstructure evolution of high $\mathrm{C}$-adopted 20Cr32Ni1 Nb alloy during different aging temperature: (a) aged at $1120{ }^{\circ} \mathrm{C}$, (b) aged at $850{ }^{\circ} \mathrm{C}$, (c) aged at $750{ }^{\circ} \mathrm{C}$, (d) phase constitution after aging Fig. 10 EDX map analyzing result of high C 20Cr32Ni1Nb alloy after aging at $850^{\circ} \mathrm{C}$ for 18 hours

Fig. 11 The $750{ }^{\circ} \mathrm{C}$ tensile test results of the high $\mathrm{C}$-adopted 20Cr32Ni1Nb alloy: (a) Strength, (b) Ductility

Fig. 12 Morphology of the alloy fracture surface: (a) cast alloy, (b) $1120{ }^{\circ} \mathrm{C}$ aged alloy, (c) $850{ }^{\circ} \mathrm{C}$ aged alloy, (d) $750{ }^{\circ} \mathrm{C}$ aged alloy

Fig. 13 Cross-section microstructure of the fracture subsurface: (a) cast alloy, (b) $1120{ }^{\circ} \mathrm{C}$ aged alloy, (c) $850{ }^{\circ} \mathrm{C}$ aged alloy, (d) $750{ }^{\circ} \mathrm{C}$ aged alloy 